\title{
Contributor Index
}

\section{Volume 42, 1998}

Subject index on page 1518.

\section{A}

Abati A (see Sgrignoli et al). 1998;42:818-820 (Letter) Abdul-Karim FW (see Cheng et al). 1998;42:1091-1094 Abdul-Karim FW (see Koscick et al). 1998;42:697-702 Abe M (see Shinagawa et al). 1998;42:407-412 Adhikari P (see Shenoi et al). 1998;42:815-816 (Letter) Afonso JL (see Vega et al). 1998;42:832-833 (Letter) Agarwal R, Khanna D, Barthwal SP. Microfilariae in a Cytologic Smear from Cavernous Hemangioma of the Liver: A Case Report. 1998;42:781-782

Agarwal S (see Mishra and Agarwal). 1998;42:11551158

Agdigos R (see Howell et al). 1998;42:171-177

Akagi A (see Takahashi et al). 1998;42:185-188

Åkerman M. New Diagnostic Techniques Applicable to Fine Needle Aspirates. 1998;42:1315-1316

Akutagawa K (see Masuda et al). 1998;42:803-806

Akutsu S (see Ishi et al). 1998;42:745-750

Ali SZ (see Allen et al). 1998;42:449-451 (Letter)

Ali SZ (see Pritchett and Ali). 1998;42:446-448 (Letter)

Ali SZ, Erozan YS. Thymoma: Cytopathologic Features and Differential Diagnosis on Fine Needle Aspiration. 1998;42:845-854

Al-Jassar AK (see Woyke et al). 1998;42:1184-1188

Al-Jazzaf H (see Woyke et al). 1998;42:1184-1188

Allen EA, Clark DP, Ali SZ. Primary Hepatic Undifferentiated Embryonal Sarcoma: Cytopathologic Findings in Peritoneal Washings. 1998;42: 449-451 (Letter)

Alonso P (see Vanda et al). 1998;42:939-944

Alonso S (see López-Ríos et al). 1998;42:796-798

Al-Rabah NA (see Kapila et al). 1998;42:1480-1481 (Letter)

Altavilla G (see Boccato et al). 1998;42:888-898

Alvaro-Naranjo T (see Bosch-Príncep et al). 1998; 42:1062-1064 (Letter)

Amma NS (see Krieger et al). 1998;42:133-140

Amma NS (see Schenck et al). 1998;42:5-15

Amma NS (see Solomon et al). 1998;42:16-24
Antica PDM (see Dominis et al). 1998;42:1053-1056 (Letter)

Araujo RWB (see Schmitt and Araujo). 1998;42: 1067-1069 (Letter)

Ardito G (see Fadda et al). 1998;42:998-1002

Argyres MI, Porter J, Rizeq MN. Diagnosis of Clinically Unsuspected Gallbladder Rupture by Peritoneal Fluid Cytology: A Case Report. 1998; 42:973-977

Arora VK (see Bhatia et al). 1998;42:865-868

Arreola JL (see Vanda et al). 1998;42:939-944

Ascoli V, Barsotti P. AgNOR Evaluation in Pulmonary Aspiration Cytology. 1998;42:1301-1302 (Letter)

Aulicino MR, Szporn AH, Dembitzer R, Mechanick J, Batheja N, Bleiweiss IJ, Burstein DE. Cytologic Findings in the Differential Diagnosis of C-Cell Hyperplasia and Medullary Carcinoma by Fine Needle Aspiration: A Case Report. 1998;42:963-967

Aurelho de Lima M, Schmitt FCdL. Expression of Dipeptidyl Aminopeptidase IV in Human Thyroid Tissues. 1998;42:445-446 (Letter)

Austin RM (see Frable et al). 1998;42:76-132

Austin RM, Ramzy I. Increased Detection of Epithelial Cell Abnormalities by Liquid-Based Gynecologic Cytology Preparations: A Review of Accumulated Data. 1998;42:178-184

Autillo-Touati A, Joannes M, d'Ercole C, RobagliaSchlupp A, Lambert A, Mazzella E, Blanc B, Seite R. HPV Typing by in Situ Hybridization on Cervical Cytologic Smears with ASCUS. 1998;42: 631-638

Ayers LW (see Sosolik et al). 1998;42:377-383

B

Baba N, Kawabata H, Kanno T, Ueno T, Makuuchi M, Morishita Y, Mori S, Sasaki J, Soejima K. Preparation of Heat-Labile Antigens on Air-Dried Cy- 
tologic Specimens for Immunocytochemical Analysis. 1998;42:707-715

Baloch Z (see Boudousquie and Baloch). 1998;42: 1485-1487 (Letter)

Barker EA. Estimating the Costs of Emerging Cytologic Technologies. 1998;42:1070-1072 (Letter)

Barsotti P (see Ascoli and Barsotti). 1998;42:13011302 (Letter)

Bartels PH, Bibbo M, Hutchinson ML, Gahm T, Grohs HK, Gwi-Mak E, Kaufman EA, Kaufman RH, Knight BK, Koss LG, Magruder LE, Mango LJ, McCallum SM, Melamed MR, Peebles A, Richart RM, Robinowitz M, Rosenthal DL, Sauer T, Schenck U, Tanaka N, Topalidis T, Verhest AP, Wertlake PT, Whittaker JA, Wilbur DC. Computerized Screening Devices and Performance Assessment: Development of a Policy Towards Automation: IAC Task Force Summary. 1998;42:59-68

Barthwal SP (see Agarwal et al). 1998;42:781-782

Batheja N (see Aulicino et al). 1998;42:963-967

Baughman RP (see Harlamert et al). 1998;42:1382-1388

Beck S (see van Ballegooijen et al). 1998;42:1133-1138

Bejarano PA (see Harlamert et al). 1998;42:1382-1388

Belk TI (see Howell et al). 1998;42:171-177

Bell DA (see Tambouret et al). 1998;42:1403-1408

Beotra A (see Chinoy et al). 1998;42:811-812 (Letter)

Bevilacqua RG (see Cerqueira et al). 1998;42:639-649

Bhagwati SN (see Shah et al). 1998;42:1149-1154

Bharadwaj R (see Krishnamurthy and Bharadwaj). 1998;42:1444-1446

Bhatia A, Singh N, Arora VK, Gupta K. Prospective Peer Review in Fine Needle Aspiration Cytology: Another Step Toward Quality Assurance. 1998; 42:865-868

Bhattathiri NV, Bindu L, Remani P, Chandralekha B, Nair KM. Radiation-Induced Acute Immediate Nuclear Abnormalities in Oral Cancer Cells: Serial Cytologic Evaluation. 1998;42:1084-1090

Bhuiya TA (see Putti et al). 1998;42:387-390

Bibbo M (see Bartels et al). 1998;42:59-68

Bibbo M (see Zimmerman and Bibbo). 1998;42:367-370

Bigner SH (see Bishop et al). 1998;42:189-197 [erratum 1998;42:12A (March-April issue)]

Bindu L (see Bhattathiri et al). 1998;42:1084-1090

Bishop JW, Bigner SH, Colgan TJ, Husain M, Howell LP, McIntosh KM, Taylor DA, Sadeghi MH. Multicenter Masked Evaluation of AutoCyte PREP Thin Layers with Matched Conventional Smears: Including Initial Biopsy Results. 1998;42: 189-197 [erratum 1998;42:12A (March-April issue)] Blanc B (see Autillo-Touati et al). 1998;42:631-638 Blandamura S (see Boccato et al). 1998;42:888-898
Bleiweiss IJ (see Aulicino et al). 1998;42:963-967

Blumenfeld W, Hashmi N, Sagerman P. Comparison of Aspiration, Touch and Scrape Preparations Simultaneously Obtained from Surgically Excised Specimens: Effect of Different Methods of Smear Preparation on Interpretive Cytologic Features. 1998;42:1414-1418

Bober P (see Saleh et al). 1998;42:1330-1335

Bobhate SK (see Kate et al). 1998;42:679-684

Boccato P, Altavilla G, Blandamura S. Fine Needle Aspiration Biopsy of Salivary Gland Lesions: A Reappraisal of Pitfalls and Problems. 1998;42:888898

Boer R (see van Ballegooijen et al). 1998;42:1133-1138

Bolick DR, Hellman DJ. Laboratory Implementation and Efficacy Assessment of the ThinPrep Cervical Cancer Screening System. 1998;42:209-213

Bolis GB, Farabi R, Liberati F, Macciò T. Lymphoepithelial Cyst of the Pancreas: Report of a Case Diagnosed by Fine Needle Aspiration Biopsy. 1998;42:384-386

Boon ME (see van Ballegooijen et al). 1998;42:1133-1138

Boran MD-S, Hilaire PJdS, Leveugle-Pin J, Patricot LM, Thivolet-Béjui F. Fine Needle Aspiration Cytology of a Solid Papillary Carcinoma of the Breast: A Case Report with Immunohistochemical Studies. 1998;42:725-728

Bosch-Príncep R, Salvadó-Usach MT, MartínezGonzàlez S, Alvaro-Naranjo T. Xanthogranulomatous Pyelonephritis: Urinary and Fine Needle Aspiration Cytology. 1998;42:1062-1064 (Letter)

Bosman C (see Di Stefano et al). 1998;42:346-356

Boudousquie AC, Baloch Z. Fine Needle Aspiration Cytology and Histopathology of Crystalloids in a Cystic Lesion of Parotid Salivary Gland. 1998;42: 1485-1487 (Letter)

Bourquin PM (see Drachenberg et al). 1998;42:10031010

Boyd JH (see Collins et al). 1998;42:1325-1329

Branca M, Morosini P, Duca P, Verderio P, Giovagnoli MR, Riti MG, Leoncini $L$, Members of the Working Group for External Quality Control in Cervical Cytopathology. Reliability and Accuracy in Reporting CIN in 14 Laboratories: Developing New Indices of Diagnostic Variability in an Interlaboratory Study. 1998;42:1370-1376

Brody JP (see Vadmal et al). 1998;42:374-376

Brugal G (see O'Brien et al). 1998;42:148-164

Brun G (see Belleannèe et al). 1998;42:945-948

Brun JL (see Belleannèe et al). 1998;42:945-948

Burke KC (see Drachenberg et al). 1998;42:1003-1010

Burstein DE (see Aulicino et al). 1998;42:963-967 


\section{C}

Cajulis RS (see Frauchiger et al). 1998;42:1139-1143

Calvez V (see Mansour et al). 1998;42:371-373

Campana A (see Vassilakos et al). 1998;42:198-202

Čáp J (see Ryška and Čáp). 1998;42:826-827 (Letter)

Capelli A (see Fadda et al). 1998;42:998-1002

Çeliktaş M (see Ersöz et al). 1998;42:799-802

Cenacchi G (see Isimbaldi et al). 1998;42:1189-1194

Cerqueira EMM, Santoro CL, Donozo NF, Freitas

BA, Pereira CAdB, Bevilacqua RG, Machado-

Santelli GM. Genetic Damage in Exfoliated Cells

of the Uterine Cervix: Association and Interaction

Between Cigarette Smoking and Progression to Malignant Transformation? 1998;42:639-649

Cha I (see Sudilovsky and Cha). 1998;42:1341-1346

Chai C (see Dodd et al). 1998;42:754-758

Chandralekha B (see Bhattathiri et al). 1998;42:10841090

Chang T-C, Tung C-C, Hsiao Y-L, Chen M-H. Immunoperoxidase Staining in the Differential Diagnosis of Parathyroid from Thyroid Origin in Fine Needle Aspirates of Suspected Parathyroid Lesions. 1998;42:619-624

Chang T-W (see Lee et al). 1998;42:391-395

Charlotte F (see Mansour et al). 1998;42:371-373

Chaturvedi S (see Mohan et al). 1998;42:772-774

Chen M-H (see Chang et al). 1998;42:619-624

Cheng L (see Lee et al). 1998;42:391-395

Cheng L, Wolf NG, Rose PG, Rodriguez M, AbdulKarim FW. Peritoneal Washing Cytology of Ovarian Tumors of Low Malignant Potential: Correlation with Surface Ovarian Involvement and Peritoneal Implants. 1998;42:1091-1094

Chern JH. AgNOR Evaluation in Pulmonary Aspiration Cytology. 1998;42:1301-1302 (Letter reply)

Chiaramonte C (see Galindo et al). 1998;42:987-990

Chinoy RF, Beotra A, Naresh KN. Fibromatosis of the Breast: Diagnosis by Fine Needle Aspiration Cytology. 1998;42:811-812 (Letter)

Chitale AR (see Shah et al). 1998;42:1149-1154

Christen H (see O'Brien et al). 1998;42:148-164

Claren R (see Isimbaldi et al). 1998;42:1189-1194

Clark DP (see Allen et al). 1998;42:449-451 (Letter)

Clark DP (see Piller and Clark). 1998;42:913-917

Claros I, de Santamaría IS, Triviño A. Violinist's or Musician's Nodule? 1998;42:833-834 (Letter)

Clément N (see Mainguene et al). 1998;42:1045-1046 (Letter)

Cobb CJ (see Niewiadomski et al). 1998;42:1060-1061 (Letter)
Cochand-Priollet B (see Krieger et al). 1998;42:133-140

Cochand-Priollet B (see McGoogan et al). 1998;42:25-32

Cochran LM (see Drachenberg et al). 1998;42:10031010

Colgan TJ (see Bishop et al). 1998;42:189-197 [erratum 1998;42:12A (March-April issue)]

Colgan TJ (see Krieger et al). 1998;42:133-140

Colgan TJ (see McGoogan et al). 1998;42:25-32

Colgan TJ (see Wilbur et al). 1998;42:214-220

Collins BT (see Skoog et al). 1998;42:1336-1340

Collins BT, Cramer HM, Ramos RR. Fine Needle Aspiration Biopsy of Recurrent and Metastatic Osteosarcoma. 1998;42:357-361

Collins BT, Gardner LJ, Verma AK, Lowe VJ, Dunphy FR, Boyd JH. Correlation of Fine Needle Aspiration Biopsy and Fluoride-18 Fluorodeoxyglucose Positron Emission Tomography in the Assessment of Locally Recurrent and Metastatic Head and Neck Neoplasia. 1998;42:1325-1329

Collins BT, Ramos RR, Grosso LE. Combined Fine Needle Aspiration Biopsy, and Immunophenotypic and Genotypic Approach to Posttransplantation Lymphoproliferative Disorders. 1998;42:869-874

Collins RJ (see Frable et al). 1998;42:76-132

Collins RJ (see Ng et al). 1998;42:1022-1026

Collins RJ (see Schenck et al). 1998;42:5-15

Collins RJ (see Solomon et al). 1998;42:16-24

Commins DL, Hinton DR. Cytologic Features of Hemangioblastoma: Comparison with Meningioma, Anaplastic Astrocytoma and Renal Cell Carcinoma. 1998;42:1104-1110

Copeland LJ (see Proca et al). 1998;42:978-982

Coppolillo EF, Peluffo M. HIV-Seropositive Pregnant Women: A High-Risk Group for Cervical SIL. 1998;42:1058-1059 (Letter)

Correa E (see Hallmann et al). 1998;42:1042-1043 (Letter)

Cosentino L (see Di Stefano et al). 1998;42:346-356

Costa MJ (see Galvez et al). 1998;42:663-667

Cotelo AM (see Mera and Cotelo). 1998;42:13041306 (Letter)

Cramer HM (see Collins et al). 1998;42:357-361

Cramer HM (see Wu et al). 1998;42:1352-1358

Cribiù FM (see Isimbaldi et al). 1998;42:1189-1194

Cubie HA (see McGoogan et al). 1998;42:1079-1083

Cuiñas C (see García-Riego et al). 1998;42:1116-1120

Dargent JL, Deplace J, Schneider E, Morales J, Kentos A, Willemart S, Roufosse C, Verhest A. Hepatocellular Carcinoma Metastatic to the Parotid 
Gland: Initial Diagnosis by Fine Needle Aspiration Biopsy. 1998;42:824-826 (Letter)

Das DK, Jain S, Tripathi RP, Parkash S, Khan IU, Rajwanshi A, Gupta S. Marginal Vacuoles in Thyroid Aspirates. 1998;42:1121-1128

Davey DD (see Krieger et al). 1998;42:133-140

Davey DD (see McGoogan et al). 1998;42:25-32

Davey DD (see Solomon et al). 1998;42:1-4

Davey DD (see Solomon et al). 1998;42:16-24

Davey DD (see Vooijs et al). 1998;42:141-147

Davi F (see Mansour et al). 1998;42:371-373

Dávila RM, Mira MC, Smith ME. Role of Cytopathology in the Diagnosis of Ocular Malignancies. 1998;42:362-366

Davis R (see Galvez et al). 1998;42:663-667

Davis RL (see Howell et al). 1998;42:171-177

Dawson AE, Mulford DK. Fine Needle Aspiration of Mucinous (Colloid) Breast Carcinoma: Nuclear Grading and Mammographic and Cytologic Findings. 1998;42:668-672

de Agustín PP (see López-Ríos et al). 1998;42:742-744 de Agustín PP (see López-Ríos et al). 1998;42:796-798 de Belleannèe G, Brun JL, Trouette H, Mompart JP, Goussot JF, Brun G, de Mascarel A. Cytologic Findings in a Neovagina Created with Vecchietti's Technique for Treating Vaginal Aplasia. 1998; 42:945-948

de Buen N (see Vanda et al). 1998;42:939-944

De Frias DVS (see Frauchiger et al). 1998;42:1139-1143

de la Vega M, Rey A, Afonso JL. Fine Needle Aspiration of Mucocelelike Lesions: Differential Diagnosis with Colloid Carcinoma. 1998;42:832-833(Letter)

de las Morenas A (see Mulligan et al). 1998;42:928-932

de los Heros CA (see Veiga et al). 1998;42:1489-1490 (Letter)

de Mascarel A (see Belleannèe et al). 1998;42:945-948

de Santamaría IS (see Claros et al). 1998;42:833-834 (Letter)

Delpiano C (see Isimbaldi et al). 1998;42:1189-1194

Dembitzer R (see Aulicino et al). 1998;42:963-967

Deplace J (see Dargent et al). 1998;42:824-826 (Letter)

d'Ercole C (see Autillo-Touati et al). 1998;42:631-638

Dharan M, Nachtigal D, Rosen G, Honigman J, Luboshitzky R. Basement Membrane Material (Hyaline Globules) in Synovial Sarcoma: A Case Report with Description of a Potential Diagnostic Pitfall. 1998;42:791-795

Di Stefano D, Scucchi LF, Cosentino L, Bosman C, Vecchione A. Intraoperative Diagnosis of Nervous System Lesions. 1998;42:346-356

Diehl ARS, Prolla JC. Rapid Rescreening of Cervical Smears for Internal Quality Control. 1998;42:949-953
DiNisco S (see Montes et al). 1998;42:396-398

DiTomasso JP (see Heintz et al). 1998;42:1431-1436

Djamin RS, Drent M, Schreurs AJM, Groen EAH, Wagenaar SS. Diagnosis of Pneumocystis carinii Pneumonia in HIV-Positive Patients: Bronchoalveolar Lavage vs. Bronchial Brushing. 1998; 42:933-938

Dodd LG, Chai C, McAdams HP, Layfield LJ. Fine Needle Aspiration of Osteogenic Sarcoma Metastatic to the Lung: A Report of Four Cases. 1998; 42:754-758

Dodd LG, Tello J. Cytologic Examination of Urine from Patients with Interstitial Cystitis. 1998;42:923-927

Domanski HA (see Persson and Domanski). 1998;42: 1066-1067 (Letter)

Dominis M, Džebro S, Kušić B, Antica PDM. Inflammatory Pseudotumor of the Spleen. 1998;42: 1053-1056 (Letter)

Donozo NF (see Cerqueira et al). 1998;42:639-649

Drachenberg CB, Bourquin PM, Cochran LM, Burke KC, Kumar D, White CS, Papadimitriou JC. Fine Needle Aspiration Biopsy of Solitary Fibrous Tumors: Report of Two Cases with Histologic, Immunohistochemical and Ultrastructural Correlation. 1998;42:1003-1010

Drent M (see Djamin et al). 1998;42:933-938

Dry S (see Montes et al). 1998;42:396-398

Duca P (see Branca et al). 1998;42:1370-1376

Ducatman BS (see Wang and Ducatman). 1998;42: 285-289

Dunphy FR (see Collins et al). 1998;42:1325-1329

Dunton CJ (see van Niekerk et al). 1998;42:33-49

Džebro S (see Dominis et al). 1998;42:1053-1056 (Letter)

\section{E}

Egawa H (see Toda et al). 1998;42:775-780

Elsheikh TM (see Wu et al). 1998;42:1352-1358

Erozan YS (see Ali and Erozan). 1998;42:845-854

Ersöz C, Zorludemir Ü, Tanyeli A, Gümürdülü D, Çeliktaş M. Fine Needle Aspiration Cytology of Hepatoblastoma: A Report of Two Cases. 1998; 42:799-802

Etori F (see Morishita et al). 1998;42:759-764

Evren I (see Kabukçuoğlu et al). 1998;42:875-882

$\mathbf{F}$

Fadda G, Mulè A, Zannoni GF, Vincenzoni C, Ardito $G$, Capelli A. Fine Needle Aspiration of a Warthin-like Thyroid Tumor: Report of a Case with 
Differential Diagnostic Criteria vs. Other LymphocyteRich Thyroid Lesions. 1998;42:998-1002

Farabi R (see Bolis et al). 1998;42:384-386

Faulkner JE, Gaba CE, Powers JD, Yam LT. Diagnosis of Primary Pancreatic Lymphoma by Fine Needle Aspiration. 1998;42:834-836 (Letter)

Florentine BD (see Niewiadomski et al). 1998;42: 1060-1061 (Letter)

Frable WJ. Current State of Malpractice Litigation. 1998;42:1302-1304 (Letter reply)

Frable WJ (see Solomon et al). 1998;42:16-24

Frable WJ, Austin RM, Greening SE, Collins RJ, Hillman RL, Kobler TP, Koss LG, Mitchell H, Perey R, Rosenthal DL, Sidoti MS, Somrak TM. Medicolegal Affairs: IAC Task Force Summary.1998;42:76-132

Frankel KA. ASCUS Diagnoses. 1998;42:271-272 (Letter)

Frankel KA. Effective Communication About Breast Aspiration. 1998;42:438-439 (Letter)

Frauchiger WL, De Frias DVS, Cajulis RS, Yu GH. The Immediate Postconization Endocervical Smear: Evaluation of Its Utility in the Detection of Residual Dysplasia. 1998;42:1139-1143

Freitas BA (see Cerqueira et al). 1998;42:639-649

Fresno MF (see Veiga et al). 1998;42:1489-1490 (Letter)

Frist S (see Solomon and Frist). 1998;42:253-259

Froberg K (see Gilcrease et al). 1998;42:1389-1396

Fujiwara K (see Minato et al). 1998;42:1455-1457

Fujiyama J (see Ueno et al). 1998;42:1424-1430

Fukuda T, Saito M, Nakajima T. Imprint Cytology of Chondroblastoma of Bone: A Case Report. 1998;42:403-406

Fukui I (see Kojima et al). 1998;42:1365-1369

Furuta T (see Kimura et al). 1998;42:817-818 (Letter)

Futter N (see Kapasi et al). 1998;42:1419-1423

\section{G}

Gaba CE (see Faulkner et al). 1998;42:834-836 (Letter) Gabriel S (see Mainguene et al). 1998;42:1045-1046 (Letter)

Gahm T (see Bartels et al). 1998;42:59-68

Gahm T (see O'Brien et al). 1998;42:148-164

Galindo LM, Soans S, Chiaramonte C, Garcia FU. Focal Hematopoietic Hyperplasia of the Rib: Report of a Case Diagnosed by Fine Needle Aspiration. 1998;42:987-990

Galvanek E (see Montes et al). 1998;42:396-398

Galvez J, Howell L, Costa MJ, Davis R. Diagnostic Concordance of Telecytology and Conventional Cytology for Evaluating Breast Aspirates. 1998; 42:663-667
Gammon RR (see Sosolik et al). 1998;42:377-383

Garcia FU (see Galindo et al). 1998;42:987-990

García-Riego A, Cuiñas C, Vilanova JJ, Ibarrola R. Extramedullary Hematopoietic Effusions. 1998; 42:1116-1120

Gardner LJ (see Collins et al). 1998;42:1325-1329

Garner D (see Melamed et al). 1998;42:69-75

Geyer JW (see Krieger et al). 1998;42:133-140

Ghougassian DF (see Raisanen et al). 1998;42:907-912

Gilbert CF (see Smith et al). 1998;42:1011-1016

Gilcrease MZ, Guzman-Paz M, Froberg K, Pambuccian S. Salivary Duct Carcinoma: Is a Specific Diagnosis Possible by Fine Needle Aspiration Cytology? 1998;42:1389-1396

Gilcrease MZ, Sahin M, Perri RT, Nelson FS, GuzmanPaz M. Fine Needle Aspiration of Signet-Ring Cell Lymphoma: A Case Report with Differential Diagnostic Considerations. 1998;42:1461-1467

Giovagnoli MR (see Branca et al). 1998;42:1370-1376

Glick T (see Gottschalk-Sabag et al). 1998;42:691-696

Gogas J (see Ioakim-Liossi et al). 1998;42:918-922

Goldstein ML, Whitman T, Renshaw AA. Significance of Cell Groups in Voided Urine. 1998;42: 290-294

Gong G (see Kim et al). 1998;42:1027-1031

González-Palacios F (see López-Ríos et al). 1998;42: 742-744

Goodell RM (see Krieger et al). 1998;42:133-140

Goodell RM (see O'Brien et al). 1998;42:148-164

Goodell RM (see Vooijs et al). 1998;42:141-147

Goodman HS (see Raisanen et al). 1998;42:907-912

Gosalbez F (see Miralles et al). 1998;42:1446-1450

Gottschalk-Sabag S, Ron N, Glick T. Use of CD34 and Factor VIII To Diagnose Hepatocellular Carcinoma on Fine Needle Aspirates. 1998;42:691-696

Goussot JF (see Belleannèe et al). 1998;42:945-948

Greening SE (see Frable et al). 1998;42:76-132

Griffin S (see Vassilakos et al). 1998;42:198-202

Groen EAH (see Djamin et al). 1998;42:933-938

Grohs DH. Estimating the Costs of Emerging Cytologic Technologies. 1998;42:1070-1072 (Letter reply)

Grohs DH. Impact of Automated Technology on the Cervical Cytologic Smear: A Comparison of Cost. 1998;42:165-170

Grohs DH (see Krieger et al). 1998;42:133-140

Grohs DH (see Vooijs et al). 1998;42:141-147

Grohs HK (see Bartels et al). 1998;42:59-68

Grohs HK (see McGoogan et al). 1998;42:25-32

Grosso LE (see Collins et al). 1998;42:869-874

Grotkowski CE (see Shidham et al). 1998;42:11591166

Gümürdülü D (see Ersöz et al). 1998;42:799-802 
Gupta K (see Bhatia et al). 1998;42:865-868

Gupta MK (see Gupta et al). 1998;42:959-962

Gupta R (see Gupta et al). 1998;42:959-962

Gupta RK (see Mehrotra and Gupta). 1998;42:840841 (Letter)

Gupta S (see Das et al). 1998;42:1121-1128

Gupta S, Gupta MK, Gupta R, Mishra RS. Breast Metastasis of Cervical Carcinoma Diagnosed by Fine Needle Aspiration Cytology: A Case Report. 1998;42:959-962

Gupta SK (see Krieger et al). 1998;42:133-140

Gupta SK (see Schenck et al). 1998;42:5-15

Gurley AM (see McGoogan et al). 1998;42:25-32

Guzman-Paz M (see Gilcrease et al). 1998;42:1389_ 1396

Guzman-Paz M (see Gilcrease et al). 1998;42:1461-1467

Gwi-Mak E (see Bartels et al). 1998;42:59-68

\section{H}

Habbema JDF (see van Ballegooijen et al). 1998;42: 1133-1138

Hadary A (see Moldavsky et al). 1998;42:1050-1051 (Letter)

Hajdu SI. Acta Cytologica 40 Years Ago: Volume II, Number 2, 1958. 1998;42:605-606

Hajdu SI. Acta Cytologica 40 Years Ago: Volume II, Number 3, 1958. 1998;42:1077-1078

Hajdu SI. The Diagnosis of Thymomas. 1998;42: 843-844

Hajdu SI. The Discovery of Trichomonas vaginalis. 1998;42:1075-1075

Hajdu SI (see Vadmal et al). 1998;42:374-376

Hajdu SI (see Vadmal et al). 1998;42:837-839 (Letter)

Hajdu SI (see Vadmal et al). 1998;42:1046-1048 (Letter)

Hallmann RSR, Schneeweiss LG, Correa E, Zamora J. Fine Needle Aspiration Biopsy of Thymic Carcinoid Tumor: A Case with Immunocytochemical Correlation. 1998;42:1042-1043 (Letter)

Hamati HF (see Sebenik et al). 1998;42:1199-1203

Hameed A (see Proca et al). 1998;42:978-982

Harlamert HA, Mira J, Bejarano PA, Baughman RP, Miller MA, Whitsett JA, Yassin R. Thyroid Transcription Factor-1 and Cytokeratins 7 and 20 in Pulmonary and Breast Carcinoma. 1998;42:1382-1388

Harper CG (see Raisanen et al). 1998;42:907-912

Hasegawa K (see Hirokawa et al). 1998;42:14871489 (Letter)

Hashmi N (see Blumenfeld et al). 1998;42:1414-1418

Heintz PW, Schmidt WA, Pommier RF, Vetto JT, DiTomasso JP. Submandibular Gland Carcinoma ex Pleomorphic Adenoma: Report of a Case with
Cytologic Features and Diagnostic Pitfalls. 1998; 42:1431-1436

Heller DS (see Schlosshauer et al). 1998;42:10431045 (Letter)

Hellman DJ (see Bolick and Hellman). 1998;42:209-213

Herbert A (see Schenck et al). 1998;42:5-15

Hijazi Y (see Sgrignoli et al). 1998;42:818-820 (Letter)

Hilaire PJds (see Boran et al). 1998;42:725-728

Hilgarth M (see van Niekerk et al). 1998;42:33-49

Hillman RL (see Frable et al). 1998;42:76-132

Hino Y (see Hirokawa et al). 1998;42:1487-1489 (Letter)

Hinton DR (see Commins and Hinton). 1998;42: 1104-1110

Hiroi M (see Takahashi et al). 1998;42:734-741

Hirokawa M (see Minato et al). 1998;42:1455-1457

Hirokawa M (see Sugihara et al). 1998;42:751-753

Hirokawa M (see Terayama et al). 1998;42:1458-1460

Hirokawa M, Monobe Y, Shimizu M, Terayama K,

Kanahara T, Manabe T. Sclerosing Lipogranuloma of the Scrotum: Report of a Case with Fine Needle Aspiration Biopsy Findings. 1998;42: 1181-1183

Hirokawa M, Sakurai T, Shimizu M, Manabe T, Kanahara T. Cytology of Pigmented Renal Cell Carcinoma: A Case Report. 1998;42:788-790

Hirokawa M, Shimizu M, Kanahara T, Manabe T. Plant Cells Mimicking Adenocarcinoma in Sputum. 1998;42:1306-1307 (Letter)

Hirokawa M, Shimizu M, Kanahara T, Mikami Y, Manabe T, Hasegawa K, Hino Y. Imprint Cytology of Lipoma with Chondroosseous Metaplasia. 1998;42:1487-1489 (Letter)

Hirokawa M, Shimizu M, Terayama K, Kanahara T, Sonoo H, Manabe T. Macrofollicular Variant of Papillary Thyroid Carcinoma: Report of a Case with Fine Needle Aspiration Biopsy Findings. 1998;42:1441-1443

Hirschowitz SL (see Lieu et al). 1998;42:1195-1198

Hofman P (see Mainguene et al). 1998;42:1045-1046 (Letter)

Honigman J (see Dharan et al). 1998;42:791-795

Hoshino T (see Shinagawa et al). 1998;42:407-412

Howat AJ. Uniform Approach to Breast Fine Needle Aspiration Biopsy. 1998;42:440 (Letter)

Howell L (see Galvez et al). 1998;42:663-667

Howell LP (see Bishop et al). 1998;42:189-197 [erratum 1998;42:12A (March-April issue)]

Howell LP, Davis RL, Belk TI, Agdigos R, Lowe J. The AutoCyte Preparation System for Gynecologic Cytology. 1998;42:171-177

Hsiao Y-L (see Chang et al). 1998;42:619-624

Huh J, Seoh JY, Kim SS. Cell Block Preparation of a 
Burkitt's Lymphoma Cell Line as a Positive Control for in Situ Hybridization for Epstein-Barr Virus. 1998;42:1144-1148

Husain M (see Bishop et al). 1998;42:189-197 [erratum 1998;42:12A (March-April issue)]

Husain OAN (see McGoogan et al). 1998;42:25-32

Hutchinson ML (see Bartels et al). 1998;42:59-68

Hutchinson ML (see McGoogan et al). 1998;42:25-32

Hutchinson ML (see Melamed et al). 1998;42:69-75

\section{I}

Ibarrola R (see García-Riego et al). 1998;42:1116-1120 Ida H (see Kobayashi et al). 1998;42:419-424

Iezumi K (see Terasaki et al). 1998;42:812-815 (Letter) Imakita M (see Masuda et al). 1998;42:803-806

Inhorn SL (see Logrono et al). 1998;42:1172-1176

Inhorn SL, Logrono R, Kurtycz DF. Criteria for Cytologic Reporting of Breast Fine Needle Aspiration. 1998;42:1482-1483 (Letter reply)

Intersociety Working Group for Cytology Technologies. A Proposed Methodology for Evaluating Secondary Screening (Rescreening) Instruments for Gynecologic Cytology. 1998;42:1311-1314

Ioakim-Liossi A, Markopoulos C, Karakitsos P, Safioleas M, Gogas J, Vaiopoulos G. p53 Protein Expression in Benign and Malignant Breast Lesions. 1998;42:918-922

Ip P (see Ng et al). 1998;42:1022-1026

Ishi K, Suzuki F, Saito A, Koyatsu J, Akutsu S, Kubota T. Cytodiagnosis of Placental Site Trophoblastic Tumor: A Report of Two Cases. 1998;42:745-750

Ishi K, Suzuki F, Saito A, Koyatsu J, Kina K, Shitoto K. Cytodiagnosis of Malignant Melanoma of Soft Tissue: Report of a Case with Diagnosis by Intraoperative Cytology. 1998;42:1177-1180

Ishi K, Suzuki F, Saito A, Koyatsu J, Kubota T. Cytodiagnosis of Vaginal Endodermal Sinus Tumor: A Case Report. 1998;42:399-402

Ishibashi-Ueda H (see Masuda et al). 1998;42:803-806 Ishida Y (see Kobayaski et al). 1998;42:1129-1132

Ishikawa Y (see Ueno et al). 1998;42:1424-1430

Ishizawa M (see Kamiya et al). 1998;42:650-656

Isimbaldi G, Santangelo M, Cenacchi G, Cribiù FM, Claren R, Delpiano C, Spinelli M. Gastrointestinal Autonomic Nerve Tumor (Plexosarcoma): Report of a Case with Fine Needle Aspiration Biopsy and Histologic, Immunocytochemical and Ultrastructural Study. 1998;42:1189-1194

Ito E, Nei H, Noda M, Saito T, Koizumi M, Kudo R. Electron Microscopic Examination of Cytologic Samples. 1998;42:1095-1103
Ito K (see Sato et al). 1998;42:342-345

Ito K (see Sato et al). 1998;42:1377-1381

Itoh H (see Satoh et al). 1998;42:413-418

Iyer VK, Kapila K, Verma K. Fine Needle Aspiration Cytology of Dermatopathic Lymphadenitis. 1998;42:1347-1351

Izu K (see Sasaki and Izu). 1998;42:829-830 (Letter)

\section{J}

Jain S (see Das et al). 1998;42:1121-1128

Jalali GR (see Kumar et al). 1998;42:1491-1492 (Letter) Jang S-J (see Paik et al). 1998;42:828-829 (Letter)

Jasso R (see Vanda et al). 1998;42:939-944

Jayaram G, Pathmanathan R, Khanijow V. Cystic Lesion of the Parotid Gland with Squamous Metaplasia Mistaken for Squamous Cell Carcinoma: A Case Report. 1998;42:1468-1472

Jeren T, Vince A. Cytologic and Immunoenzymatic Findings in CSF from Patients with Tick-Borne Encephalitis. 1998;42:330-334

Jiko K (see Sato et al). 1998;42:342-345

Jimenez-Ayala M (see Schenck et al). 1998;42:5-15

Jiménez-Heffernan JA (see Vicandi et al). 1998;42: 335-341

Jirousek Z (see Ryška and Jirousek). 1998;42:443-445 (Letter)

Joannes M (see Autillo-Touati et al). 1998;42:631-638 Jones BA (see Krieger et al). 1998;42:133-140

Julius CJ (see Sosolik et al). 1998;42:377-383

Junaid TA (see Kapila et al). 1998;42:1480-1481 (Letter)

K

Kabukçuoğlu F, Kabukçuoğlu Y, Kuzgun U, Evren I. Fine Needle Aspiration of Malignant Bone Lesions. 1998;42:875-882

Kabukçuoğlu Y (see Kabukçuoğlu F et al). 1998;42: 875-882

Kachi H (see Morishita et al). 1998;42:759-764

Kadivar M (see Vasei et al). 1998;42:1437-1440

Kamal MM (see Kate et al). 1998;42:679-684

Kamiya M, Mizuguchi K, Yoshimoto M, Tanaka M, Motegi S, Matushima H, Ishizawa M, Nakamura K. Cytologic Diagnosis of Signet-Ring Cell Carcinoma of the Breast. 1998;42:650-656

Kanabuchi K (see Sato et al). 1998;42:1492-1494 (Letter)

Kanahara T (see Hirokawa et al). 1998;42:788-790

Kanahara T (see Hirokawa et al). 1998;42:1181-1183 
Kanahara T (see Hirokawa et al). 1998;42:1306-1307 (Letter)

Kanahara T (see Hirokawa et al). 1998;42:1441-1443

Kanahara T (see Hirokawa et al). 1998;42:1487-1489 (Letter)

Kanahara T (see Sugihara et al). 1998;42:751-753

Kanahara T (see Terayama et al). 1998;42:1458-1460

Kaneko C (see Kobayaski et al). 1998;42:1129-1132

Kaneko C, Shamoto M, Kobayashi TK. Nuclear Grooves in Vaginal Cells. 1998;42:823-824 (Letter)

Kanno T (see Baba et al). 1998;42:707-715

Kanter L (see Nilsson et al). 1998;42:1317-1324

Kapasi H, Robertson S, Futter N. Diagnosis of Renal Malacoplakia by Fine Needle Aspiration Cytology: A Case Report. 1998;42:1419-1423

Kapila K (see Iyer et al). 1998;42:1347-1351

Kapila K, Al-Rabah NA, Junaid TA. Ectopic Breast Tissue on the Vulva Diagnosed by Fine Needle Aspiration. 1998;42:1480-1481 (Letter)

Karakitsos P (see Ioakim-Liossi et al). 1998;42:918-922

Karakitsos P (see O'Brien et al). 1998;42:148-164

Karlén J (see Nilsson et al). 1998;42:1317-1324

Kase H, Kodama S, Nagai E, Tanaka K. Glutathione S-Transferase $\pi$ Immunostaining of CisplatinResistant Ovarian Cancer Cells in Ascites. 1998; 42:1397-1402

Kashimura M (see Matsuura et al). 1998;42:625-630

Kashkari S (see Vesoulis and Kashkari). 1998;42: 1032-1036

Kate MS, Kamal MM, Bobhate SK, Kher AV. Evaluation of Fine Needle Capillary Sampling in Superficial and Deep-Seated Lesions: An Analysis of 670 Cases. 1998;42:679-684

Kato H (see van Niekerk et al). 1998;42:33-49

Kato M (see Kobayashi et al). 1998;42:716-720

Katz RL (see Shin and Katz). 1998;42:855-864

Kaufman EA (see Bartels et al). 1998;42:59-68

Kaufman EA (see Melamed et al). 1998;42:69-75

Kaufman RH (see Bartels et al). 1998;42:59-68

Kaufman RH (see Richart et al). 1998;42:50-58

Kaufman RH (see van Niekerk et al). 1998;42:33-49

Kawabata H (see Baba et al). 1998;42:707-715

Kawagoe T (see Matsuura et al). 1998;42:625-630

Kawamori T (see Morishita et al). 1998;42:759-764

Kawan E, Ulrich W, Redtenbacher S, Schreiber B, Zwick H. Kawan Bronchial Brush/Cell Block Technique: Facilitation of the Routine Diagnosis of Bronchial Neoplasms. 1998;42:1409-1413

Kentos A (see Dargent et al). 1998;42:824-826 (Letter) Kerekes Z (see Ryška et al). 1998;42:826-827 (Letter) Keyhani-Rofagha S (see Proca et al). 1998;42:978-982 Khan IU (see Das et al). 1998;42:1121-1128
Khanijow V (see Jayaram et al). 1998;42:1468-1472 Khanna D (see Agarwal et al). 1998;42:781-782 Kher AV (see Kate et al). 1998;42:679-684

Kho J (see Wu et al). 1998;42:1352-1358

Khoo U (see Ng et al). 1998;42:1022-1026

Kijima H (see Sato et al). 1998;42:1492-1494 (Letter)

Kijima H (see Terasaki et al). 1998;42:812-815 (Letter)

Kilpatrick SE. Fine Needle Aspiration Biopsy of Langerhans Cell Histiocytosis of Bone: Are Ancillary Studies Necessary for a "Definitive" Diagnosis? 1998;42:820-823 (Letter)

Kim O (see Kim et al). 1998;42:1027-1031

Kim SS (see Huh et al). 1998;42:1144-1148

Kim T, Yoon GS, Kim O, Gong G. Fine Needle Aspiration Diagnosis of Malignant Mixed Tumor (Carcinosarcoma) Arising in Pleomorphic Adenoma of the Salivary Gland: A Case Report. 1998;42:1027-1031

Kimura M (see Takahashi et al). 1998;42:185-188

Kimura M, Furuta T, Teramura K, Satou T. Pseudohyphae of Cryptococcus in a Cytologic Specimen. 1998;42:817-818 (Letter)

Kina K (see Ishi et al). 1998;42:1177-1180

Kishida F (see Kuwabara et al). 1998;42:440-443 (Letter)

Kishita H (see Masuda et al). 1998;42:803-806

Kiyuna M (see Toda et al). 1998;42:775-780

Knesel EA Jr (see McGoogan et al). 1998;42:25-32

Knesel EA Jr (see O'Brien et al). 1998;42:148-164

Knesel EA Jr (see Vooijs et al). 1998;42:141-147

Knight BK (see Bartels et al). 1998;42:59-68

Knight BK (see Solomon et al). 1998;42:16-24

Ko J-S (see Tsou et al). 1998;42:1359-1364

Ko YH (see Oh et al). 1998;42:1167-1171

Kobayashi TK (see Kaneko et al). 1998;42:823-824 (Letter)

Kobayashi TK, Sugihara H, Kato M, Watanabe S. Cytologic Features of Granulomatous Mastitis: Report of a Case with Fine Needle Aspiration Cytology and Immunocytochemical Findings. 1998; 42:716-720

Kobayashi TK, Tamagaki T, Yoneyama C, Watanabe S, Sugihara H, Ida H. Imprint Cytology of Gaucher's Disease Presenting as a Splenic Mass: A Case Report with Molecular Approaches. 1998;42:419-424

Kobayaski TK, Tsubota K, Kaneko C, Ishida Y. BarShaped Nuclear Chromatin in Conjunctival Samples: With Cytologic Features and Ultrastructural Correlation. 1998;42:1129-1132

Kobilková J (see Schenck et al). 1998;42:5-15

Kobler T (see O’Brien et al). 1998;42:148-164 
Kobler TP (see Frable et al). 1998;42:76-132

Kobler TP (see Melamed et al). 1998;42:69-75

Kodama S (see Kase et al). 1998;42:1397-1402

Koduru P (see Vadmal et al). 1998;42:374-376

Kohno I (see Minato et al). 1998;42:1455-1457

Kohno K (see Kuwabara et al). 1998;42:440-443 (Letter)

Koizumi M (see Ito et al). 1998;42:1095-1103

Kojima Si, Sekine H, Fukui I, Ohshima H. Clinical Significance of "Cannibalism" in Urinary Cytology of Bladder Cancer. 1998;42:1365-1369

Konno R (see Sato et al). 1998;42:295-298

Konno R (see Sato et al). 1998;42:299-304

Konno R (see Sato et al). 1998;42:342-345

Konno R (see Sato et al). 1998;42:1377-1381

Konno R (see Sato et al). 1998;42:1483-1485 (Letter)

Koscick RL, Petersilge CA, Makley JT, AbdulKarim FW. CT-Guided Fine Needle Aspiration and Needle Core Biopsy of Skeletal Lesions: Complementary Diagnostic Techniques. 1998;42: 697-702

Koshitaka Y (see Shinagawa et al). 1998;42:407-412

Koss LG (see Bartels et al). 1998;42:59-68

Koss LG (see Frable et al). 1998;42:76-132

Koss LG (see Krieger et al). 1998;42:133-140

Koulos JP (see Schlosshauer et al). 1998;42:1043-1045 (Letter)

Kouno S (see Shinagawa et al). 1998;42:407-412

Koyatsu J (see Ishi et al). 1998;42:399-402

Koyatsu J (see Ishi et al). 1998;42:745-750

Koyatsu J (see Ishi et al). 1998;42:1177-1180

Krane JF, Renshaw AA. Relative Value and CostEffectiveness of Culture and Special Stains in Fine Needle Aspirates of the Lung. 1998;42:305-311

Kreicbergs A (see Nilsson et al). 1998;42:1317-1324

Krieger PA (see Melamed et al). 1998;42:69-75

Krieger PA, McGoogan E, Vooijs GP, Amma NS, Coch-Priollet B, Colgan TJ, Davey DD, Geyer JW, Goodell RM, Grohs DH, Gupta SK, Jones BA, Koss LG, Mango LJ, McCallum SM, Nielsen M, Robinowitz M, Sauer T, Schumann JL, Syrjänen KJ, Suprun HZ, Topalidis T, Wertlake PT, Whittaker J. Quality Assurance/Control Issues: IAC Task Force Summary. 1998;42:133-140

Krishnamurthy S, Bharadwaj R. Fine Needle Aspiration Cytology of Clear Cell Sarcoma of the Kidney: A Case Report. 1998;42:1444-1446

Kuan L (see Lee et al). 1998;42:221-226

Kubota T (see Ishi et al). 1998;42:399-402

Kubota T (see Ishi et al). 1998;42:745-750

Kudo R (see Ito et al). 1998;42:1095-1103

Kuhel WI, Kutler DI, Santos-Buch CA. Poorly Differentiated Insular Thyroid Carcinoma: A Case
Report with Identification of Intact Insulae with Fine Needle Aspiration Biopsy. 1998;42:991-997

Kumar D (see Drachenberg et al). 1998;42:1003-1010

Kumar PV. Testicular Leukemia Relapse: Fine Needle Aspiration Findings. 1998;42:312-316

Kumar PV (see Vasei et al). 1998;42:1437-1440

Kumar PV, Jalali GR, Noorani H. Acute Myelomonocytic Leukemia Initially Presenting as Bilateral Renal Enlargement and Diagnosed by FNAC. 1998;42:1491-1492 (Letter)

Kumar PV, Monabati A. Bronchial Oncocytoma Diagnosed by Brushing Cytology. 1998;42:10561057 (Letter)

Kumar PV, Monabati A. Nipplelike Nuclear Protrusions in Conjunctival Cells. 1998;42:1057-1058 (Letter)

Kumarasinghe MP. Cytomorphologic Features of Diffuse Sclerosing Variant of Papillary Carcinoma of the Thyroid: A Report of Two Cases in Children. 1998;42:983-986

Kurtycz DF (see Inhorn et al). 1998;42:1481-1481 (Letter reply)

Kurtycz DF (see Logrono et al). 1998;42:1172-1176

Kušić B (see Dominis et al). 1998;42:1053-1056 (Letter)

Kutler DI (see Kuhel et al). 1998;42:991-997

Kuwabara H, Kohno K, Kishida F, Uda H, Miyabe K, Nagao K, Saito K, Shibanushi T. Imprint Cytology of Malignant Plasmacytoid Myoepithelioma of the Palate. 1998;42:440-443 (Letter)

Kuzgun U (see Kabukçuoğlu et al). 1998;42:875-882

Kyrkou KA (see O’Brien et al). 1998;42:148-164

\section{$\mathbf{L}$}

Labate AM (see Vadmal et al). 1998;42:837-839 (Letter)

Labbe S (see O'Brien et al). 1998;42:148-164

Laforga JBM, López JI. Breast Carcinoma with Endocrine Features: Report of a Case with Cytologic and Immunohistochemical Studies. 1998;42:1017-1021

Lambert A (see Autillo-Touati et al). 1998;42:631-638

Larsson O (see Nilsson et al). 1998;42:1317-1324

Layfield LJ (see Dodd et al). 1998;42:754-758

Layfield LJ, Mooney E. Fine Needle Aspiration Cytology of Poroid Hidradenoma. 1998;42:10641066 (Letter)

Lebovics R (see Sgrignoli et al). 1998;42:818-820 (Letter)

Lee J-D (see Paik et al). 1998;42:828-829 (Letter)

Lee JSJ, Kuan L, Oh S, Patten FW, Wilbur DC. A Feasibility Study of the AutoPap System LocationGuided Screening. 1998;42:221-226 
Lee WY, Cheng L, Chang T-W. Fine Needle Aspiration Cytology of Malignant Phyllodes Tumor with Liposarcomatous Stroma of the Breast: A Case Report. 1998;42:391-395

Lehmann U, Poche-de Vos F, Poche-Blohm I. Detection of HPV DNA in Archival, PapanicolaouStained Cervical Smears Using PCR. 1998;42: 1051-1053 (Letter)

Leoncini L (see Branca et al). 1998;42:1370-1376

Leveugle-Pin J (see Boran et al). 1998;42:725-728

Liberati F (see Bolis et al). 1998;42:384-386

Lieu D, Hirschowitz SL, Skinner KA, Zuckerbraun L. Recurrent Secondary Hyperparathyroidism After Autotransplantation into the Sternocleidomastoid Muscle: Report of a Case with Fine Needle Aspiration Findings. 1998;42:1195-1198

Lin K-J (see Tsou et al). 1998;42:1359-1364

Lin Y-M (see Tsou et al). 1998;42:1359-1364

Linder J (see McGoogan et al). 1998;42:25-32

Logrono R (see Inhorn et al). 1998;42:1481 (Letter reply)

Logrono R, Kurtycz DF, Wojtowycz M, Inhorn SL. Fine Needle Aspiration Cytology of Fibrous Dysplasia: A Case Report. 1998;42:1172-1176

Long EL (see O'Brien et al). 1998;42:148-164

López JI (see Laforga and López). 1998;42:1017-1021

López JI, Rodil MA. Fine Needle Aspiration Cytology of an Umbilical Cyst Mimicking Sister Mary Joseph's Nodule. 1998;42:1069-1070 (Letter)

López JI, Rodil MA. Fine Needle Aspiration Cytology of the Cutaneous Manifestation of Primary Sinonasal Plasmacytoma. 1998;42:839-840 (Letter)

López-Ferrer P (see Vicandi et al). 1998;42:335-341

López-Ríos F, Alonso S, Lozano F, Agustín PP. Metastatic Squamous Cell Carcinoma in a Liver Graft: Report of a Case Diagnosed by Fine Needle Aspiration. 1998;42:796-798

López-Ríos F, Vargas-Castrillón J, GonzálezPalacios F, de Agustín PP. Breast Carcinoma in Situ in a Male: Report of a Case Diagnosed by Nipple Discharge Cytology. 1998;42:742-744

Loubière R (see Mainguene et al). 1998;42:1045-1046 (Letter)

Lowe J (see Howell et al). 1998;42:171-177

Lowe VJ (see Collins et al). 1998;42:1325-1329

Lozano F (see López-Ríos et al). 1998;42:796-798

Lozano MD, Panizo A, Sola IJ, Pardo-Mindán FJ. FNAC Guided by Computed Tomography in the Diagnosis of Primary Pancreatic Adenosquamous Carcinoma: A Report of Three Cases. 1998;42:1451-1454
Luboshitzky R (see Dharan et al). 1998;42:791-795

Luff RD (see Solomon et al). 1998;42:16-24

\section{M}

Macciò T (see Bolis et al). 1998;42:384-386

Machado-Santelli GM (see Cerqueira et al). 1998;42: 639-649

Madge R (see Renshaw et al). 1998;42:1111-1115

Madrigal B (see Veiga et al). 1998;42:1489-1490 (Letter)

Magruder LE (see Bartels et al). 1998;42:59-68

Mainguene C, Clément N, Gabriel S, Loubière R, Hofman P. Urogenital Schistosomiasis: An Unusual Discovery on Cervical Smears from a Caucasian Female. 1998;42:1045-1046 (Letter)

Makino H (see Sato et al). 1998;42:836-837 (Letter)

Makley JT (see Koscick et al). 1998;42:697-702

Makuuchi M (see Baba et al). 1998;42:707-715

Malekhoseini SA (see Vasei et al). 1998;42:1437-1440

Manabe T (see Hirokawa et al). 1998;42:788-790

Manabe T (see Hirokawa et al). 1998;42:1181-1183

Manabe T (see Hirokawa et al). 1998;42:1306-1307 (Letter)

Manabe T (see Hirokawa et al). 1998;42:1441-1443

Manabe T (see Hirokawa et al). 1998;42:1487-1489

(Letter)

Manabe T (see Minato et al). 1998;42:1455-1457

Manabe T (see Sugihara et al). 1998;42:751-753

Manabe T (see Terayama et al). 1998;42:1458-1460

Mango LJ (see Bartels et al). 1998;42:59-68

Mango LJ (see Krieger et al). 1998;42:133-140

Mango LJ (see McGoogan et al). 1998;42:25-32

Mango LJ (see O’Brien et al). 1998;42:148-164

Mango LJ (see Radensky and Mango). 1998;42:246-252

Mango LJ (see van Niekerk et al). 1998;42:33-49

Mango LJ (see Vooijs et al). 1998;42:141-147

Mango LJ, Radensky PW. Interactive Neural Network-Assisted Screening: A Clinical Assessment. 1998;42:233-245

Mango LJ, Valente PT. Neural Network-Assisted Analysis and Microscopic Rescreening in Presumed Negative Cervical Cytologic Smears: A Comparison. 1998;42:227-232

Manjon J-A (see Miralles et al). 1998;42:1446-1450

Mansour G, Charlotte F, Calvez V, Davi F, MerleBéral H. AIDS-Related Primary Lymphoma of the Pleural Cavity: A Case Report. 1998;42:371-373

Markopoulos C (see Ioakim-Liossi et al). 1998;42: 918-922

Martínez-Gonzàlez S (see Bosch-Príncep et al). 1998;42:1062-1064 (Letter)

Maruta J (see Sato et al). 1998;42:1377-1381 
Maruta J (see Sato et al). 1998;42:1483-1485 (Letter)

Masood S. Fine Needle Aspiration of the Breast: A Practical Approach to Diagnosis of Carcinoma. 1998;42:283-284

Masood S (see Richart et al). 1998;42:50-58

Masuda K, Akutagawa K, Yutani C, Kishita H, Ishibashi-Ueda H, Imakita M. Persistent Infection with Human Polyomavirus Revealed by Urinary Cytology in a Patient with Heart Transplantation: A Case Report. 1998;42:803-806

Matsunaga G (see Sato et al). 1998;42:295-298

Matsunaga G (see Sato et al). 1998;42:299-304

Matsunaga G (see Sato et al). 1998;42:836-837 (Letter)

Matsuura Y, Kawagoe T, Toki N, Sugihara K, Kashimura M. Low Grade Cervical Intraepithelial Neoplasia Associated with Human Papillomavirus Infection: Long-Term Follow-up. 1998; 42:625-630

Matushima H (see Kamiya et al). 1998;42:650-656

Mazzella E (see Autillo-Touati et al). 1998;42:631-638

McAdams HP (see Dodd et al). 1998;42:754-758

McCallum SM (see Bartels et al). 1998;42:59-68

McCallum SM (see Krieger et al). 1998;42:133-140

McGoogan E (see Krieger et al). 1998;42:133-140

McGoogan E (see O'Brien et al). 1998;42:148-164

McGoogan E, Colgan TJ, Ramzy I, Cochand-Priollet B, Davey DD, Grohs HK, Gurley AM, Husain OAN, Hutchinson ML, Knesel EA Jr, Linder J, Mango LJ, Mitchell H, Peebles A, Reith A, Robinowitz M, Sauer T, Shida S, Solomon D, Topalidis T, Wilbur DC, Yamauchi K. Cell Preparation Methods and Criteria for Sample Adequacy: IAC Task Force Summary. 1998;42:25-32

McGoogan E, Seagar AL, Cubie HA. Detection of High-Risk Human Papillomavirus Nucleic Acid in Archival Cervical Smears. 1998;42:1079-1083

McIntosh KM (see Bishop et al). 1998;42:189-197 [erratum 1998;42:12A (March-April issue)]

Mechanick J (see Aulicino et al). 1998;42:963-967

Medalie NS, Vallejo CE, Wasserman P. Metastatic Pulmonary Artery Sarcoma: Report of a Case with Diagnosis by Fine Needle Aspiration. 1998; 42:968-972

Medley G (see Mitchell and Medley). 1998;42:260-264 Medley G (see Mitchell and Medley). 1998;42:265-270

Megevand E (see Vassilakos et al). 1998;42:198-202

Mehrotra R, Gupta RK, Gupta RK. Microfilariae of Wuchereria bancrofti Identified in a Cervicovaginal Smear. 1998;42:840-841 (Letter)

Meisels A (see Richart et al). 1998;42:50-58

Meisels A (see Solomon et al). 1998;42:16-24

Melamed MR (see Bartels et al). 1998;42:59-68
Melamed MR, Hutchinson ML, Kaufman EA, Schechter CB, Garner D, Kobler TP, Krieger PA, Reith A, Schenck U. Evaluation of Costs and Benefits of Advances in Cytologic Technology: IAC Task Force Summary. 1998;42:69-75

The Members of the Working Group for External Quality Control in Cervical Cytopathology (see Branca et al). 1998;42:1370-1376

Mendoza N (see Vela et al). 1998;42:954-958

MenendezP (see Miralles et al). 1998;42:1446-1450

Mera AdlC (see Schmitt and Mera). 1998;42:721-724

Mera AdlC, Cotelo AM. Breast Metastases. 1998; 42:1304-1306 (Letter)

Merle-Béral H (see Mansour et al). 1998;42:371-373

Mikami Y (see Hirokawa et al). 1998;42:1487-1489 (Letter)

Miller MA (see Harlamert et al). 1998;42:1382-1388

Miller WM (see Wilbur et al). 1998;42:214-220

Minato H, Shimizu M, Hirokawa M, Fujiwara K, Kohno I, Manabe T. Adenocarcinoma in Situ of the Fallopian Tube: A Case Report. 1998;42:1455-1457

Mira J (see Harlamert et al). 1998;42:1382-1388

Miralles TG, Gosalbez F, Menendez P, Manjon J-A, Sampedro A. Fine Needle Aspiration of Sclerosing Lymphocytic Lobulitis of the Breast: A Report of Two Cases. 1998;42:1446-1450

Miranda MC (see Dávila et al). 1998;42:362-366

Mirdha BR. Echinococcus oligarthrus. 1998;42: 1309-1310 (Letter)

Mirdha BR. Necrosis and Charcot-Leyden Crystals. 1998;42:1310-1310 (Letter)

Mishra K, Agarwal S. Fine Needle Aspiration Cytology of Malignant Chondroid Syringoma: A Case Report. 1998;42:1155-1158

Mishra RS (see Gupta et al). 1998;42:959-962

Misra PK (see Mohan et al). 1998;42:772-774

Mitchell H (see Frable et al). 1998;42:76-132

Mitchell H (see McGoogan et al). 1998;42:25-32

Mitchell H, Medley G. Detection of Laboratory False Negative Smears by the PAPNET Cytologic Screening System. 1998;42:265-270

Mitchell H, Medley G. Detection of Unsuspected Abnormalities by PAPNET-Assisted Review. 1998;42:260-264

Miyabe K (see Kuwabara et al). 1998;42:440-443 (Letter)

Miyajima Y (see Satoh et al). 1998;42:413-418

Mizuguchi K (see Kamiya et al). 1998;42:650-656

Mohan G, Chaturvedi S, Misra PK. Microfilaria in a Fine Needle Aspirate of Primary Solid Malignant Tumor of the Maxillary Antrum: A Case Report. 1998;42:772-774 
Mohite JD (see Pandit et al). 1998;42:1307-1309 (Letter)

Moldavsky M, Hadary A, Turani H. Xanthogranulomatous Cholecystitis. 1998;42:1050-1051 (Letter)

Mompart JP (see Belleannèe et al). 1998;42:945-948

Monabati A (see Kumar and Monabati). 1998;42: 1056-1057 (Letter)

Monabati A (see Kumar and Monabati). 1998;42: 1057-1058 (Letter)

Monobe Y (see Hirokawa et al). 1998;42:1181-1183

Montes MA, DiNisco S, Dry S, Galvanek E. Fine Needle Aspiration Cytology of Primary Isolated Splenic Blastomyces dermatitidis: A Case Report. 1998;42:396-398

Mooney E (see Layfield and Mooney). 1998;42:10641066 (Letter)

Morales J (see Dargent et al). 1998;42:824-826 (Letter)

Mori S (see Baba et al). 1998;42:707-715

Moriki T (see Takahashi et al). 1998;42:734-741

Morishita Y (see Baba et al). 1998;42:707-715

Morishita Y, Etori F, Sawada K, Kachi H, Yamada T, Kawamori T, Tanaka T. Sarcomatous Hepatocellular Carcinoma with Malignant Ascites: A Report of Two Cases. 1998;42:759-764

Morosini P (see Branca et al). 1998;42:1370-1376

Mosquera J-A (see Ribas and Mosquera). 1998;42: 685-690

Motegi S (see Kamiya et al). 1998;42:650-656

Mui KK (see Vooijs et al). 1998;42:141-147

Mulè A (see Fadda et al). 1998;42:998-1002

Mulford DK (see Dawson and Mulford). 1998;42: 668-672

Müller HA (see Peters et al). 1998;42:1048-1050 (Letter)

Mulligan NJ, de las Morenas A, Soto-Wright V, O'Brien MJ. Percentages of Cervical Cytologic Diagnoses as a Quality Assurance Method. 1998;42: 928-932

Muzumdar GA (see Shah et al). 1998;42:1149-1154

\section{$\mathbf{N}$}

Nachtigal D (see Dharan et al). 1998;42:791-795

Nadimpalli V (see Thinakaran et al). 1998;42:769-771

Nagai E (see Kase et al). 1998;42:1397-1402

Nagao K (see Kuwabara et al). 1998;42:440-443 (Letter)

Nair KM (see Bhattathiri et al). 1998;42:1084-1090

Nair SG (see Sebenik et al). 1998;42:1199-1203

Naitoh M (see Takahashi et al). 1998;42:185-188

Nakajima T (see Fukuda et al). 1998;42:403-406

Nakamura K (see Kamiya et al). 1998;42:650-656

Nakayama H (see Takahashi et al). 1998;42:734-741

Nandi GK (see Shenoi et al). 1998;42:815-816 (Letter)

Naresh KN (see Chinoy et al). 1998;42:811-812 (Letter)
Navin J (see Solomon et al). 1998;42:16-24

Nawaz S (see Singh and Nawaz). 1998;42:437-438 (Letter)

Nayak VM (see Sahoo et al). 1998;42:435-436 (Letter)

Nei H (see Ito et al). 1998;42:1095-1103

Nelson FS (see Gilcrease et al). 1998;42:1461-1467

$\mathrm{Ng}$ H-K. Smears in the Diagnosis of Pituitary Adenomas. 1998;42:614-618

Ng H-K (see Teo and Ng). 1998;42:673-678

Ng WK, Khoo U, Ip P, Collins RJ. Fine Needle Aspiration Cytology of Myxopapillary Ependymoma: A Case Report. 1998;42:1022-1026

Nielsen M (see Krieger et al). 1998;42:133-140

Nielsen M (see Schenck et al). 1998;42:5-15

Nielsen ML (see Vooijs et al). 1998;42:141-147

Niewiadomski S, Florentine BD, Cobb CJ. Cytologic Identification of Trichomonas vaginalis in Urine from a Male with Long-Standing Sterile Pyuria. 1998;42:1060-1061 (Letter)

Nilsson G, Wang M, Wejde J, Kanter L, Karlén J, Tani E, Kreicbergs A, Larsson O. Reverse Transcriptase Polymerase Chain Reaction on Fine Needle Aspirates for Rapid Detection of Translocations in Synovial Sarcoma. 1998;42:1317-1324

Nishimura M (see Sangawa et al). 1998;42:425-429

Noda M (see Ito et al). 1998;42:1095-1103

Noorani H (see Kumar et al). 1998;42:1491-1492 (Letter)

Nosanchuk JS. Histologic Processing of Thick Tissue Specimens from Cytology Slides. 1998;42: 831 (Letter)

Novis DA. Current State of Malpractice Litigation. 1998;42:1302-1304 (Letter)

Nozawa S (see Solomon et al). 1998;42:1-4

Nozawa S (see van Niekerk et al). 1998;42:33-49

Oberholzer M (see O’Brien et al). 1998;42:148-164

O'Brien MJ (see Mulligan et al). 1998;42:928-932

O'Brien MJ, Takahashi M, Brugal G, Christen H, Gahm T, Goodell RM, Karakitsos P, Knesel EA Jr, Kobler T, Kyrkou KA, Labbe S, Long EL, Mango LJ, McGoogan E, Oberholzer M, Reith A, Winkler C. Digital Imagery/Telecytology: IAC Task Force Summary. 1998;42:148-164

Oh S (see Lee et al). 1998;42:221-226

Oh YL, Ko YH, Ree HJ. Aspiration Cytology of Ectopic Cervical Thymoma Mimicking a Thyroid Mass: A Case Report. 1998;42:1167-1171

Ohno T (see Ohwada et al). 1998;42:765-768

Ohshima H (see Kojima et al). 1998;42:1365-1369 
Ohwada M, Suzuki M, Ohno T, Saito K, Sato I. Appearance of Primary Endometrial and Ovarian Clear Cell Adenocarcinoma 17 Months Postpartum: A Case Report. 1998;42:765-768

Okamoto H (see Sato et al).1998;42:1492-1494 (Letter)

Olmos R (see Vanda et al). 1998;42:939-944

Olszewski WT (see Richart et al). 1998;42:50-58

Omiya H (see Terasaki et al). 1998;42:812-815 (Letter)

Ortega L (see Vicandi et al). 1998;42:335-341

Osamura RY (see Satoh et al). 1998;42:413-418

Otiniano L (see Vela et al). 1998;42:954-958

Pai RR (see Shenoi et al). 1998;42:815-816 (Letter)

Pai U (see Shenoi et al). 1998;42:815-816 (Letter)

Paik SS, Jang SJ, Park YW, Lee JD. Actinomycosis in a Lymph Node Diagnosed by Fine Needle Aspiration Cytology. 1998;42:828-829 (Letter)

Pambuccian S (see Gilcrease et al). 1998;42:1389-1396

Pandit AA, Vaideeswar P, Mohite JD. Infarction of a Thyroid Nodule After Fine Needle Aspiration Biopsy. 1998;42:1307-1309 (Letter)

Panizo A (see Lozano et al). 1998;42:1451-1454

Papadimitriou JC (see Drachenberg et al). 1998;42: 1003-1010

Papellas J (see Yang et al). 1998;42:703-706

Papillo JL, Zarka MA, St. John TL. Evaluation of the ThinPrep Pap Test in Clinical Practice: A SevenMonth, 16,314-Case Experience in Northern Vermont. 1998;42:203-208

Pardo-Mindán FJ (see Lozano et al). 1998;42:1451-1454

Park Y-W (see Paik et al). 1998;42:828-829 (Letter)

Parkash S (see Das et al). 1998;42:1121-1128

Pass H (see Sgrignoli et al). 1998;42:818-820 (Letter)

Pathmanathan R (see Jayaram et al). 1998;42:14681472

Patricot LM (see Boran et al). 1998;42:725-728

Patten FW (see Lee et al). 1998;42:221-226

Pawlick GF (see Wilbur et al). 1998;42:214-220

Peebles A (see Bartels et al). 1998;42:59-68

Peebles A (see McGoogan et al). 1998;42:25-32

Peluffo M (see Coppolillo and Peluffo). 1998;42: 1058-1059 (Letter)

Pereira CAdB (see Cerqueira et al). 1998;42:639-649

Perey R (see Frable et al). 1998;42:76-132

Pérez-Guillermo M, Sola-Pérez J. Hepatoblastomas and Undifferentiated (Embryonal) Sarcoma of the Liver: Updating References While Revising Galley Proofs. 1998;42:439-440 (Letter)

Perri RT (see Gilcrease et al). 1998;42:1461-1467

Persson PG, Domanski HA. Fine Needle Aspiration
Cytology of Uterine Leiomyosarcoma Metastatic to the Tongue. 1998;42:1066-1067 (Letter)

Peters K, Müller HA, Peters W. Cytology of Pericardial Effusion: Can Color Be a Predictor of Malignancy? 1998;42:1048-1050 (Letter)

Peters W (see Peters et al). 1998;42:1048-1050 (Letter)

Petersilge CA (see Koscick et al). 1998;42:697-702

Piller CF, Clark DP. Pathologic Outcome in HIVSeropositive Individuals with Nonspecific Bronchoalveolar Lavage Cytology. 1998;42:913-917

Pitman MB (see Tambouret et al). 1998;42:1403-1408

Poche-Blohm I (see Lehmann et al). 1998;42:10511053 (Letter)

Poche-de Vos F (see Lehmann et al). 1998;42:10511053 (Letter)

Pommier RF (see Heintz et al). 1998;42:1431-1436

Poon TP, Tchertkoff V, Win H. Subacute Measles Encephalitis with AIDS Diagnosed by Fine Needle Aspiration Biopsy: A Case Report. 1998;42: 729-733

Porter J (see Argyres et al). 1998;42:973-977

Powers JD (see Faulkner et al). 1998;42:834-836 (Letter)

Prasoon D. Breast Filariasis Mimicking Paget's Disease. 1998;42:452-453 (Letter)

Prasoon D. Follicular Carcinoma of Thyroid Gland Presenting as Scalp Metastasis. 1998;42:451-452 (Letter)

Prey MU (see Wilbur et al). 1998;42:214-220

Pritchett DD, Ali SZ. Metastatic Medullary Thyroid Carcinoma in a Breast Fine Needle Aspirate: Cytopathologic Findings. 1998;42:446-448 (Letter)

Proca D, Keyhani-Rofagha S, Copeland LJ, Hameed A. Exfoliative Cytology of Neuroendocrine Small Cell Carcinoma of the Endometrium: A Report of Two Cases. 1998;42:978-982

Prolla JC (see Diehl and Prolla). 1998;42:949-953

Putti TC, Bhuiya TA, Wasserman PG. Fine Needle Aspiration Cytology of Mixed Tall and Columnar Cell Papillary Carcinoma of the Thyroid: A Case Report. 1998;42:387-390

Quinn TJ (see Shidham et al). 1998;42:1159-1166

\section{$\mathbf{R}$}

Radensky PW (see Mango and Radensky). 1998;42: 233-245

Radensky PW, Mango LJ. Interactive Neural Network-Assisted Screening: An Economic Assessment. 1998;42:246-252 
Rai R (see Reddy et al). 1998;42:454 (Letter)

Raisanen J, Goodman HS, Ghougassian DF, Harper CG. Role of Cytology in the Intraoperative Diagnosis of Central Demyelinating Disease. 1998;42: 907-912

Rajwanshi A (see Das et al). 1998;42:1121-1128

Ramos RR (see Collins et al). 1998;42:357-361

Ramos RR (see Collins et al). 1998;42:869-874

Ramos RR (see Skoog et al). 1998;42:1336-1340

Ramzy I (see Austin and Ramsy). 1998;42:178-184

Ramzy I (see McGoogan et al). 1998;42:25-32

Reddy PS, Sabhikhi AK, Rai R. Microfilariae in Fine Needle Aspirates of the Liver. 1998;42:454 (Letter)

Redtenbacher S (see Kawan et al). 1998;42:1409-1413

Ree HJ (see Oh et al). 1998;42:1167-1171

Rege JD (see Shet and Rege). 1998;42:657-662

Reith A (see McGoogan et al). 1998;42:25-32

Reith A (see Melamed et al). 1998;42:69-75

Reith A (see O'Brien et al). 1998;42:148-164

Remani P (see Bhattathiri et al). 1998;42:1084-1090

Renshaw AA (see Goldstein et al). 1998;42:290-294

Renshaw AA (see Krane and Renshaw). 1998;42: 305-311

Renshaw AA, Madge R, Sugarbaker DJ, Swanson S. Malignant Pleural Effusions After Resection of Pulmonary Adenocarcinoma. 1998;42:11111115

Rey A (see Vega et al). 1998;42:832-833 (Letter)

Ribas A, Mosquera J-A. Ameboflagellates in Bronchial Asthma. 1998;42:685-690

Ricci JL (see Vadmal et al). 1998;42:837-839 (Letter)

Richart RM (see Bartels et al). 1998;42:59-68

Richart RM (see van Niekerk et al). 1998;42:33-49

Richart RM, Masood S, Syrjänen KJ, Vassilakos P, Kaufman RH, Meisels A, Olszewski WT, Sakamoto A, Stoler MH, Vooijs GP, Wilbur DC. Human Papillomavirus: IAC Task Force Summary. 1998;42:50-58

Riti MG (see Branca et al). 1998;42:1370-1376

Rizeq MN (see Argyres et al). 1998;42:973-977

Robaglia-Schlupp A (see Autillo-Touati et al). 1998; 42:631-638

Robertson S (see Kapasi et al). 1998;42:1419-1423

Robinowitz M (see Bartels et al). 1998;42:59-68

Robinowitz M (see Krieger et al). 1998;42:133-140

Robinowitz M (see McGoogan et al). 1998;42:25-32

Robinowitz M (see van Niekerk et al). 1998;42:33-49

Rodil MA (see López and Rodil). 1998;42:1069-1070 (Letter)

Rodil MA (see López and Rodil). 1998;42:839-840 (Letter)

Rodriguez M (see Cheng et al). 1998;42:1091-1094
Rollins SD. Criteria for Cytologic Reporting of Breast Fine Needle Aspiration. 1998;42:1482 (Letter) Ron N (see Gottschalk-Sabag et al). 1998;42:691-696 Rose PG (see Cheng et al). 1998;42:1091-1094

Rosen G (see Dharan et al). 1998;42:791-795

Rosenthal DL (see Bartels et al). 1998;42:59-68

Rosenthal DL (see Frable et al). 1998;42:76-132

Rosenthal DL (see Solomon et al). 1998;42:16-24

Roufosse C (see Dargent et al). 1998;42:824-826 (Letter)

Ryška A, Jirousek Z. Peripheral Giant Cell Granuloma: A Diagnostic Pitfall in FNAC Material. 1998;42:443-445 (Letter)

Ryška A, Kerekes Z, Cap J. Aspiration Cytology of Parathyroid Adenoma: Radioimmunoassay of Parathormone in Aspirate as a Helpful Diagnostic Tool. 1998;42:826-827 (Letter)

S

Sabhikhi AK (see Reddy et al). 1998;42:454 (Letter)

Sadeghi MH (see Bishop et al). 1998;42:189-197 [erratum 1998;42:12A (March-April issue)]

Sadi AM (see Toda et al). 1998;42:775-780

Safioleas M (see Ioakim-Liossi et al). 1998;42:918-922

Sagerman P (see Blumenfeld et al). 1998;42:1414-1418

Sahai K (see Sahoo et al). 1998;42:435-436 (Letter)

Sahin M (see Gilcrease et al). 1998;42:1461-1467

Sahoo M, Sahai K, Nayak aVM. Scapulohumeral Tuberculosis Diagnosed by Fine Needle Aspiration Cytology. 1998;42:435-436 (Letter)

Saito A (see Ishi et al). 1998;42:399-402

Saito A (see Ishi et al). 1998;42:745-750

Saito A (see Ishi et al). 1998;42:1177-1180

Saito K (see Kuwabara et al). 1998;42:440-443 (Letter)

Saito K (see Ohwada et al). 1998;42:765-768

Saito M (see Fukuda et al). 1998;42:403-406

Saito T (see Ito et al). 1998;42:1095-1103

Sakamoto A (see Richart et al). 1998;42:50-58

Sakurai T (see Hirokawa et al). 1998;42:788-790

Saleh HA, Bober P, Tabaczka P. Improved Detection of Adenocarcinoma of Serous Fluids with p53 Immunocytochemistry. 1998;42:1330-1335

Salvadó-Usach MT (see Bosch-Príncep et al). 1998; 42:1062-1064 (Letter)

Sampedro A (see Miralles et al). 1998;42:1446-1450

Sangawa A, Shintaku M, Nishimura M. Nuclear Pseudoinclusions in Angiomyolipoma of the Kidney: A Case Report. 1998;42:425-429

Santangelo M (see Isimbaldi et al). 1998;42:1189-1194

Santillán P (see Vanda et al). 1998;42:939-944

Santoro CL (see Cerqueira et al). 1998;42:639-649

Santos-Buch CA (see Kuhel et al). 1998;42:991-997 
Sasaki H (see Sato et al). 1998;42:1377-1381

Sasaki J (see Baba et al). 1998;42:707-715

Sasaki J, Izu K. Automatic Fine Needle Aspiration Pistol Specially Designed for Small Tumors. 1998;42:829-830 (Letter)

Sasano H (see Sato et al). 1998;42:342-345

Sato I (see Ohwada et al). 1998;42:765-768

Sato S (see Sato et al). 1998;42:1492-1494 (Letter)

Sato S (see Terasaki et al). 1998;42:812-815 (Letter)

Sato S, Ito K, Jiko K, Konno R, Yajima A, Sasano H. Cytologic Application of p53 Overexpression Using Immunocytochemistry and Confocal Laser Scanning Microscopy. 1998;42:342-345

Sato S, Makino H, Matsunaga G, Yajima A. False Negative Rate in Mass Screening for Cervical Cancer. 1998;42:836-837 (Letter)

Sato S, Maruta Ji, Ito K, Konno R, Yajima A, Sasaki H. Prognostic Features of Cervical Dysplasia Associated with Specific Types of HPV DNA and Cytologic Features Characteristic of HPV Infection in Dysplasia. 1998;42:1377-1381

Sato S, Maruta Ji, Konno R, Yajima A. In Situ Detection of HPV in a Cervical Smear with in Situ Hybridization. 1998;42:1483-1485 (Letter)

Sato S, Matsunaga G, Konno R, Yajima A. Mass Screening for Cancer of the Endometrium in Miyagi Prefecture, Japan. 1998;42:295-298

Sato S, Matsunaga G, Konno R, Yajima A. Mass Screening for Cancer of the Uterine Cervix in Miyagi Prefecture, Japan: Effects and Problems. 1998;42:299-304

Sato T, Sato S, Yoshida H, Terasaki Y, Okamoto H, Kanabuchi K, Kijima H. Diffuse Large B-cell Lymphoma with Sclerosis of the Thymus. 1998; 42:1492-1494 (Letter)

Satoh F, Umemura S, Itoh H, Miyajima Y, Tokuda Y, Tajima T, Osamura RY. Fine Needle Aspiration Cytology of Glycogen-Rich Clear Cell Carcinoma of the Breast: A Case Report. 1998;42:413418

Satoh Y (see Ueno et al). 1998;42:1424-1430

Satou T (see Kimura et al). 1998;42:817-818 (Letter)

Sauer T (see Bartels et al). 1998;42:59-68

Sauer T (see Krieger et al). 1998;42:133-140

Sauer T (see McGoogan et al). 1998;42:25-32

Sauer T (see Solomon et al). 1998;42:16-24

Sawada K (see Morishita et al). 1998;42:759-764

Schechter CB (see Melamed et al). 1998;42:69-75

Schenck U (see Bartels et al). 1998;42:59-68

Schenck U (see Melamed et al). 1998;42:69-75

Schenck U, Herbert A, Solomon D, Amma NS, Collins RJ, Gupta SK, Jimenez-Ayala M, Ko- bilková J, Nielsen M, Suprun HZ. Terminology: IAC Task Force Summary. 1998;42:5-15

Schlosshauer PW, Heller DS, Koulos JP. Malignant Melanoma of the Uterine Cervix Diagnosed on a Cervical Cytologic Smear. 1998;42:1043-1045 (Letter)

Schmidt WA (see Heintz et al). 1998;42:1431-1436

Schmitt FC, Araujo RWB. Cytologic Diagnosis of Ulcerative Eosinophilic Granuloma of the Tongue. 1998;42:1067-1069 (Letter)

Schmitt FC, Mera AdlC. Fine Needle Aspiration Cytology Presentation of a Cellular Variant of Breast Myofibroblastoma: Report of a Case with Immunohistochemical Studies. 1998;42:721-724

Schmitt FCdL (see Aurelho de Lima and Schmitt). 1998;42:445-446 (Letter)

Schneeweiss LG (see Hallmann et al). 1998;42:10421043 (Letter)

Schneider E (see Dargent et al). 1998;42:824-826 (Letter)

Schneider V. Hans-Klaus Zinser, M.D., F.I.A.C.: 1912-1997. 1998;42:455

Schreiber B (see Kawan et al). 1998;42:1409-1413

Schreurs AJM (see Djamin et al). 1998;42:933-938

Schumann JL (see Krieger et al). 1998;42:133-140

Scucchi LF (see Di Stefano et al). 1998;42:346-356

Seagar AL (see McGoogan et al). 1998;42:1079-1083

Sebenik M, Nair SG, Hamati HF. Primary Small Cell Anaplastic Carcinoma of the Breast Diagnosed by Fine Needle Aspiration Cytology: A Case Report. 1998;42:1199-1203

Seite R (see Autillo-Touati et al). 1998;42:631-638

Sekine H (see Kojima et al). 1998;42:1365-1369

Seoh JY (see Huh et al). 1998;42:1144-1148

Sgrignoli A, Abati A, Pass H, Lebovics R, Hijazi Y. Metastatic Mesothelioma Presenting as a Salivary Gland Neoplasm. 1998;42:818-820 (Letter)

Shah AB, Muzumdar GA, Chitale AR, Bhagwati SN. Squash Preparation and Frozen Section in Intraoperative Diagnosis of Central Nervous System Tumors. 1998;42:1149-1154

Shamoto M (see Kaneko et al). 1998;42:823-824 (Letter) Sheils LA, Wilbur DC. ASCUS Diagnoses. 1998;42: 271-272 (Letter reply)

Shenoi U, Pai RR, Pai U, Nandi GK, Adhikari P. Microfilariae in Bone Marrow Aspiration Smears. 1998;42:815-816 (Letter)

Shet TM, Rege JD. Aspiration Cytology of Tubular Adenomas of the Breast: An Analysis of Eight Cases. 1998;42:657-662

Shibanushi T (see Kuwabara et al). 1998;42:440-443 (Letter) 
Shida S (see McGoogan et al). 1998;42:25-32

Shidham VB, Weiss JP, Quinn TJ, Grotkowski CE. Fine Needle Aspiration Cytology of Gastric Solitary Fibrous Tumor: A Case Report. 1998;42: 1159-1166

Shimizu M (see Hirokawa et al). 1998;42:788-790

Shimizu M (see Hirokawa et al). 1998;42:1181-1183

Shimizu M (see Hirokawa et al). 1998;42:1306-1307 (Letter)

Shimizu M (see Hirokawa et al). 1998;42:1441-1443

Shimizu M (see Hirokawa et al). 1998;42:1487-1489 (Letter)

Shimizu M (see Minato et al). 1998;42:1455-1457

Shimizu M (see Sugihara et al). 1998;42:751-753

Shimizu M (see Terayama et al). 1998;42:1458-1460

Shin HJC, Katz RL. Thymic Neoplasia as Represented by Fine Needle Aspiration Biopsy of Anterior Mediastinal Masses: A Practical Approach to the Differential Diagnosis. 1998;42:855864

Shinagawa T, Tadokoro M, Abe M, Koshitaka Y, Kouno S, Hoshino T. Papillary Urothelial Carcinoma of the Urinary Bladder Demonstrating Prominent Signet-Ring Cells in a Smear: A Case Report. 1998;42:407-412

Shintaku M (see Sangawa et al). 1998;42:425-429

Shitoto K (see Ishi et al). 1998;42:1177-1180

Sidoti MS (see Frable et al). 1998;42:76-132

Silverman JF (see Smith et al). 1998;42:1011-1016

Simples P (see Thinakaran et al). 1998;42:769-771

Singh M, Nawaz S. Fine Needle Aspiration of Breast Hamartoma. 1998;42:437-438 (Letter)

Singh N (see Bhatia et al). 1998;42:865-868

Singh SK (see Thinakaran et al). 1998;42:769-771

Skinner KA (see Lieu et al). 1998;42:1195-1198

Skoog L, Collins BT, Tani E, Ramos RR. Fine Needle Aspiration Cytology of Penile Tumors. 1998;42: 1336-1340

Smilari TF (see Vadmal et al). 1998;42:374-376

Smilari TF (see Vadmal et al). 1998;42:1046-1048 (Letter)

Smith AR, Gilbert CF, Strausbauch P, Silverman JF. Fine Needle Aspiration Cytology of a Mediastinal Granular Cell Tumor with Histologic Confirmation and Ancillary Studies: A Case Report. 1998;42:1011-1016

Smith ME (see Dávila et al). 1998;42:362-366

Soans S (see Galindo et al). 1998;42:987-990

Soejima K (see Baba et al). 1998;42:707-715

Sola IJ (see Lozano et al). 1998;42:1451-1454

Sola-Pérez J (see Pérez-Guillermo and Sola-Pérez). 1998;42:439-440 (Letter)
Solomon D (see McGoogan et al). 1998;42:25-32

Solomon D (see Schenck et al). 1998;42:5-15

Solomon D, Davey DD, Nozawa S, Syrjänen KJ. Editorial: Future Directions in Cervical Cytology. 1998;42:1-4

Solomon D, Frable WJ, Vooijs GP, Wilbur DC, Amma NS, Collins RJ, Davey DD, Knight BK, Luff RD, Meisels A, Navin J, Rosenthal DL, Sauer T, Stoler M, Suprun HZ, Yamauchi K. ASCUS and AGUS Criteria: IAC Task Force Summary. 1998;42:16-24

Solomon HM, Frist S. PAPNET Testing for HSILs: The Few Cell/Small Cell Challenge. 1998;42:253259

Somrak TM (see Frable et al). 1998;42:76-132

Somrak TM (see Vooijs et al). 1998;42:141-147

Sonoo H (see Hirokawa et al). 1998;42:1441-1443

Sosolik RC, Gammon RR, Julius CJ, Ayers LW. Pulmonary Alveolar Proteinosis: A Report of Two Cases with Diagnostic Features in Bronchoalveolar Lavage Specimens. 1998;42:377-383

Soto-Wright V (see Mulligan et al). 1998;42:928-932 Spinelli M (see Isimbaldi et al). 1998;42:1189-1194

St. John TL (see Papillo et al). 1998;42:203-208

Stoler M (see Solomon et al). 1998;42:16-24

Stoler MH (see Richart et al). 1998;42:50-58

Strausbauch P (see Smith et al). 1998;42:1011-1016

Sudilovsky D, Cha I. Fine Needle Aspiration Cytology of Dermatopathic Lymphadenitis. 1998;42: 1341-1346

Sugarbaker DJ (see Renshaw et al). 1998;42:1111-1115

Sugihara H (see Kobayashi et al). 1998;42:419-424

Sugihara H (see Kobayashi et al). 1998;42:716-720

Sugihara K (see Matsuura et al). 1998;42:625-630

Sugihara K, Hirokawa M, Shimizu M, Manabe T, Kanahara T. Collagenous Crystalloids in a Fine Needle Aspirate of a Pleomorphic Adenoma of the Minor Salivary Gland: A Case Report. 1998; 42:751-753

Suprun HZ (see Krieger et al). 1998;42:133-140 Suprun HZ (see Schenck et al). 1998;42:5-15

Suprun HZ (see Solomon et al). 1998;42:16-24

Suto A (see Terasaki et al). 1998;42:812-815 (Letter)

Suzuki F (see Ishi et al). 1998;42:399-402

Suzuki F (see Ishi et al). 1998;42:745-750

Suzuki F (see Ishi et al). 1998;42:1177-1180

Suzuki M (see Ohwada et al). 1998;42:765-768

Swanson S (see Renshaw et al). 1998;42:1111-1115

Syrjänen KJ (see Krieger et al). 1998;42:133-140

Syrjänen KJ (see Richart et al). 1998;42:50-58

Syrjänen KJ (see Solomon et al). 1998;42:1-4

Szporn AH (see Aulicino et al). 1998;42:963-967 


\section{$\mathbf{T}$}

Tabaczka P (see Saleh et al). 1998;42:1330-1335

Tadokoro M (see Shinagawa et al). 1998;42:407-412

Tajima T (see Satoh et al). 1998;42:413-418

Takahashi M (see O'Brien et al). 1998;42:148-164

Takahashi M, Kimura M, Akagi A, Naitoh M. Auto-

Cyte SCREEN Interactive Automated Primary

Cytology Screening System: A Preliminary Evaluation. 1998;42:185-188

Takahashi T, Moriki T, Hiroi M, Nakayama H. Invasive Lobular Carcinoma of the Breast with Osteoclastlike Giant Cells: A Case Report. 1998;42: 734-741

Takeshita T (see Terasaki et al). 1998;42:812-815 (Letter)

Tamagaki T (see Kobayashi et al). 1998;42:419-424

Tamamoto T (see Toda et al). 1998;42:775-780

Tambouret R, Pitman MB, Bell DA. Benign Glandular Cells in Posthysterectomy Vaginal Smears. 1998;42:1403-1408

Tanaka K (see Kase et al). 1998;42:1397-1402

Tanaka M (see Kamiya et al). 1998;42:650-656

Tanaka N (see Bartels et al). 1998;42:59-68

Tanaka T (see Morishita et al). 1998;42:759-764

Tani E (see Nilsson et al). 1998;42:1317-1324

Tani E (see Skoog et al). 1998;42:1336-1340

Tanyeli A (see Ersöz et al). 1998;42:799-802

Taylor DA (see Bishop et al). 1998;42:189-197 [erratum 1998;42:12A (March-April issue)]

Tchertkoff V (see Poon et al). 1998;42:729-733

Tello J (see Dodd and Tello). 1998;42:923-927

Teo JG-C, Ng H-K. Cytodiagnosis of Pilocytic Astrocytoma in Smear Preparations.1998;42:673-678

Teramura K (see Kimura et al). 1998;42:817-818 (Letter)

Terasaki Y (see Sato et al).1998;42:1492-1494 (Letter)

Terasaki Y, Sato S, Yoshida H, Iezumi K, Suto CTA, Takeshita T, Omiya H, Kijima H. Invasive Lobular Breast Carcinoma Mimicking Medullary Carcinoma. 1998;42:812-815 (Letter)

Terayama K (see Hirokawa et al). 1998;42:1181-1183

Terayama K (see Hirokawa et al). 1998;42:1441-1443

Terayama K, Hirokawa M, Shimizu M, Kanahara T, Manabe T. Sertoli Cell Tumor of the Testis: Report of a Case with Imprint Cytology Findings. 1998;42:1458-1460

Thinakaran V, Singh SK, Simples P, Nadimpalli V. Fine Needle Aspiration Diagnosis of Pilomatrixoma: A Case Report. 1998;42:769-771

Thivolet-Béjui F (see Boran et al). 1998;42:725-728

Toda T, Sadi AM, Kiyuna M, Egawa H, Tamamoto T, Toyoda Z. Pigmented Neuroectodermal
Tumor of Infancy in the Epididymis: A Case Report. 1998;42:775-780

Toki N (see Matsuura et al). 1998;42:625-630

Tokuda Y (see Satoh et al). 1998;42:413-418

Topalidis T (see Bartels et al). 1998;42:59-68

Topalidis T (see Krieger et al). 1998;42:133-140

Topalidis T (see McGoogan et al). 1998;42:25-32

Toyoda Z (see Toda et al). 1998;42:775-780

Tripathi RP (see Das et al). 1998;42:1121-1128

Triviño A (see Claros et al). 1998;42:833-834 (Letter)

Trouette H (see Belleannèe et al). 1998;42:945-948

Tsou MH, Lin YM, Lin KJ, Ko JS, Wu ML. Fine Needle Aspiration Cytodiagnosis of Liver Tumors: Results Obtained with Riu's Stain. 1998;42:1359-1364 Tsubota K (see Kobayaski et al). 1998;42:1129-1132 Tung C-C (see Chang et al). 1998;42:619-624

Turani H (see Moldavsky et al). 1998;42:1050-1051 (Letter)

\section{U}

Uchiyama T (see Ueno et al). 1998;42:1424-1430

Uda H (see Kuwabara et al). 1998;42:440-443 (Letter)

Ueno M, Fujiyama J, Yamazaki I, Uchiyama T, Ishikawa Y, Satoh Y. Cytology of Primary Pulmonary Meningioma: Report of the First Multiple Case. 1998;42:1424-1430

Ueno T (see Baba et al). 1998;42:707-715

Ulrich W (see Kawan et al). 1998;42:1409-1413

Umemura S (see Satoh et al). 1998;42:413-418

\section{V}

Vadmal MS, Labate AM, Hajdu SI, Ricci JL. Primary Amyloidoma (Amyloid Tumor) of Soft Tissue. 1998;42:837-839 (Letter)

Vadmal MS, Smilari TF, Brody JP, Koduru P, Hajdu SI. Cytodiagnosis of a Primary Effusion Lymphoma: A Case Report. 1998;42:374-376

Vadmal MS, Smilari TF, Hajdu SI. Fine Needle Aspiration Features of a Metastatic Hepatocellular Carcinoma Presenting as a Symptomatic Bony Lesion. 1998;42:1046-1048 (Letter)

Vaideeswar P (see Pandit et al). 1998;42:1307-1309 (Letter)

Vaiopoulos G (see Ioakim-Liossi et al). 1998;42:918-922

Valente PT (see Mango and Valente). 1998;42: 227-232

Valero G (see Vanda et al). 1998;42:939-944

Vallejo CE (see Medalie et al). 1998;42:968-972

van Ballegooijen $\mathrm{M}$, Beck S, Boon ME, Boer R, Habbema JDF. Rescreen Effect in Conventional 
and PAPNET Screening Observed in a Study Using Material Enriched with Positive Smears. 1998;42:1133-1138

van Niekerk WA, Dunton CJ, Richart RM, Hilgarth M, Kato H, Kaufman RH, Mango LJ, Nozawa S, Robinowitz M. Colposcopy, Cervicography, Speculoscopy and Endoscopy: IAC Task Force Summary. 1998;42:33-49

Vanda B, de Buen N, Jasso R, Valero G, Vargas MH, Olmos R, Arreola JL, Santillán P, Alonso P. Inflammatory Cells and Ferruginous Bodies in Bronchoalveolar Lavage in Urban Dogs. 1998;42: 939-944

Vargas MH (see Vanda et al). 1998;42:939-944

Vargas-Castrillón J (see López-Ríos et al). 1998;42: 742-744

Vasei M, Kumar PV, Malekhoseini SA, Kadivar M. Papillary Hürthle Cell Carcinoma (Warthin-like Tumor) of the Thyroid: Report of a Case with Fine Needle Aspiration Findings. 1998;42:1437-1440

Vassilakos P. Management of Suboptimal Cytologic Smears: Persistent Inflammatory Smears. 1998; 42:1481 (Letter)

Vassilakos P (see Richart et al). 1998;42:50-58

Vassilakos P, Griffin S, Megev E, Campana A. CytoRich Liquid-Based Cervical Cytologic Test: Screening Results in a Routine Cytopathology Service. 1998;42:198-202

Vecchione A (see Di Stefano et al). 1998;42:346-356

Veiga M, Fresno MF, Madrigal B, Heros CAdl. Pleomorphic Lipoma on Fine Needle Aspiration Cytology. 1998;42:1489-1490 (Letter)

Vela C, Mendoza N, Otiniano L. Cytologic Diagnosis of Chlamydia in Cervicovaginal Secretions: Use of a Papanicolaou Stain Modification with Buffered Wright Solution. 1998;42:954-958

Verbeek DH. Histologic Processing of Thick Tissue Specimens from Cytology Slides. 1998;42:831-831 (Letter reply)

Verderio P (see Branca et al). 1998;42:1370-1376

Verhest A (see Dargent et al). 1998;42:824-826 (Letter)

Verhest AP (see Bartels et al). 1998;42:59-68

Verma AK (see Collins et al). 1998;42:1325-1329

Verma K (see Iyer et al). 1998;42:1347-1351

Vesoulis Z. Metastatic Laryngeal Basaloid Squamous Cell Carcinoma Simulating Primary Small Cell Carcinoma of the Lung on Fine Needle Aspiration Lung Biopsy: A Case Report. 1998;42:783-787

Vesoulis Z, Kashkari S. Fine Needle Aspiration of Secretory Breast Carcinoma Resembling Lactational Changes: A Case Report. 1998;42:1032-1036

Vetto JT (see Heintz et al). 1998;42:1431-1436
Vicandi B, Jiménez-Heffernan JA, López-Ferrer P, Ortega L, Viguer JM. Nodular Pseudoangiomatous Stromal Hyperplasia of the Breast: Cytologic Features. 1998;42:335-341

Viguer JM (see Vicandi et al). 1998;42:335-341

Vilanova JJ (see García-Riego et al). 1998;42:1116-1120

Vince A (see Jeren and Vince). 1998;42:330-334

Vincenzoni C (see Fadda et al). 1998;42:998-1002

Vooijs GP (see Krieger et al). 1998;42:133-140

Vooijs GP (see Richart et al). 1998;42:50-58

Vooijs GP (see Solomon et al). 1998;42:16-24

Vooijs GP, Davey DD, Somrak TM, Goodell RM, Grohs DH, Knesel EA Jr, Mango LJ, Mui KK, Nielsen ML, Wilbur DC. Computerized Training and Proficiency Testing: IAC Task Force Summary. 1998;42:141-147

W

Wagenaar SS (see Djamin et al). 1998;42:933-938

Waisman J (see Yang et al). 1998;42:703-706

Wan LS (see Yang et al). 1998;42:703-706

Wang HH, Ducatman BS. Fine Needle Aspiration of the Breast: A Probabilistic Approach to Diagnosis of Carcinoma. 1998;42:285-289

Wang M (see Nilsson et al). 1998;42:1317-1324

Wasserman P (see Medalie et al). 1998;42:968-972

Wasserman PG (see Putti et al). 1998;42:387-390

Watanabe S (see Kobayashi et al). 1998;42:419-424

Watanabe S (see Kobayashi et al). 1998;42:716-720

Weiss JP (see Shidham et al). 1998;42:1159-1166

Wejde J (see Nilsson et al). 1998;42:1317-1324

Wen JW (see Zaharopoulos et al). 1998;42:317-329 [erratum 1998;42:1493]

Wen JW (see Zaharopoulos et al). 1998;42:607-613

Wertlake PT (see Bartels et al). 1998;42:59-68

Wertlake PT (see Krieger et al). 1998;42:133-140

White CS (see Drachenberg et al). 1998;42:1003-1010

Whitman T (see Goldstein et al). 1998;42:290-294

Whitsett JA (see Harlamert et al). 1998;42:1382-1388

Whittaker J (see Krieger et al). 1998;42:133-140

Whittaker JA (see Bartels et al). 1998;42:59-68

Wilbur DC (see Bartels et al). 1998;42:59-68

Wilbur DC (see Lee et al). 1998;42:221-226

Wilbur DC (see McGoogan et al). 1998;42:25-32

Wilbur DC (see Richart et al). 1998;42:50-58

Wilbur DC (see Sheils and Wilbur). 1998;42:271-272 (Letter reply)

Wilbur DC (see Solomon et al). 1998;42:16-24

Wilbur DC (see Vooijs et al). 1998;42:141-147

Wilbur DC, Prey MU, Miller WM, Pawlick GF, Colgan TJ. The AutoPap System for Primary Screen- 
ing in Cervical Cytology: Comparing the Results of a Prospective, Intended-Use Study with Routine Manual Practice. 1998;42:214-220

Willemart S (see Dargent et al). 1998;42:824-826 (Letter)

Win H (see Poon et al). 1998;42:729-733

Winkler C (see O'Brien et al). 1998;42:148-164

Wojtowycz M (see Logrono et al). 1998;42:1172-1176

Wolf NG (see Cheng et al). 1998;42:1091-1094

Wong J (see Zaharopoulos et al). 1998;42:317-329 [erratum 1998;42:1493]

Wong J (see Zaharopoulos et al). 1998;42:607-613

Woyke S, Al-Jassar AK, Al-Jazzaf H. Macrofollicular Variant of Papillary Thyroid Carcinoma Diagnosed by Fine Needle Aspiration Biopsy: A Case Report. 1998;42:1184-1188

Wu HHJ, Cramer HM, Kho J, Elsheikh TM. Fine Needle Aspiration Cytology of Benign Adrenal Cortical Nodules: A Comparison of Cytologic Findings with Those of Primary and Metastatic Adrenal Malignancies. 1998;42:1352-1358

Wu M-L (see Tsou et al). 1998;42:1359-1364

\section{Y}

Yajima A (see Sato et al). 1998;42:295-298

Yajima A (see Sato et al). 1998;42:299-304

Yajima A (see Sato et al). 1998;42:342-345

Yajima A (see Sato et al). 1998;42:836-837 (Letter)

Yajima A (see Sato et al). 1998;42:1377-1381

Yajima A (see Sato et al). 1998;42:1483-1485 (Letter)

Yam LT (see Faulkner et al). 1998;42:834-836 (Letter)

Yamada T (see Morishita et al). 1998;42:759-764

Yamauchi K (see McGoogan et al). 1998;42:25-32

Yamauchi K (see Solomon et al). 1998;42:16-24

Yamazaki I (see Ueno et al). 1998;42:1424-1430

Yang GCH, Wan LS, Papellas J, Waisman J. Com- pact Cell Blocks: Use for Body Fluids, Fine Needle Aspirations and Endometrial Brush Biopsies. 1998;42:703-706

Yassin R (see Harlamert et al). 1998;42:1382-1388

Yoneyama C (see Kobayashi et al). 1998;42:419-424

Yoon GS (see Kim et al). 1998;42:1027-1031

Yoshida H (see Sato et al). 1998;42:1492-1494 (Letter)

Yoshida H (see Terasaki et al). 1998;42:812-815 (Letter)

Yoshimoto M (see Kamiya et al). 1998;42:650-656

Yu GH (see Frauchiger et al). 1998;42:1139-1143

Yutani C (see Masuda et al). 1998;42:803-806

\section{Z}

Zaharopoulos P, Wen JW, Wong J. Membranous Lamellar Cytoplasmic Inclusions in Histiocytes and Mesothelial Cells of Serous Fluids: Their Relationship to Phagocytosis of Red Blood Cells. 1998;42:607-613

Zaharopoulos P, Wong J, Wen JW. Nuclear Protrusions in Cells from Cytologic Specimens: Mechanisms of Formation. 1998;42:317-329 [erratum 1998;42:1493]

Zamora J (see Hallmann et al). 1998;42:1042-1043 (Letter)

Zannoni GF (see Fadda et al). 1998;42:998-1002

Zardawi IM. Fine Needle Aspiration Cytology in a Rural Setting. 1998;42:899-906

Zardawi IM. Fine Needle Aspiration Cytology vs. Core Biopsy in a Rural Setting. 1998;42:883-887

Zarka MA (see Papillo et al). 1998;42:203-208

Zimmerman RL, Bibbo M. Fine Needle Aspiration Diagnosis of a Pulmonary Metastasis from a $\mathrm{Cu}-$ taneous Adenoid Cystic Carcinoma: A Case Report. 1998;42:367-370

Zorludemir Ü (see Ersöz et al). 1998;42:799-802

Zuckerbraun L (see Lieu et al). 1998;42:1195-1198

Zwick H (see Kawan et al). 1998;42:1409-1413 